\title{
Indication of a Differential Freeze-Out in Proton-Proton and Heavy-Ion Collisions at RHIC and LHC Energies
}

\author{
Dhananjaya Thakur, ${ }^{1}$ Sushanta Tripathy, ${ }^{1}$ Prakhar Garg, \\ Raghunath Sahoo, ${ }^{1}$ and Jean Cleymans ${ }^{2}$ \\ ${ }^{1}$ Discipline of Physics, School of Basic Sciences, Indian Institute of Technology Indore, Khandwa Road, Simrol, \\ Madhya Pradesh 453552, India \\ ${ }^{2}$ UCT-CERN Research Centre and Department of Physics, University of Cape Town, Rondebosch 7701, South Africa \\ Correspondence should be addressed to Raghunath Sahoo; raghunath.sahoo@cern.ch
}

Received 12 August 2016; Accepted 3 October 2016

Academic Editor: Andrea Coccaro

Copyright (C) 2016 Dhananjaya Thakur et al. This is an open access article distributed under the Creative Commons Attribution License, which permits unrestricted use, distribution, and reproduction in any medium, provided the original work is properly cited. The publication of this article was funded by SCOAP ${ }^{3}$.

\begin{abstract}
The experimental data from the RHIC and LHC experiments of invariant $p_{T}$ spectra for most peripheral $A+A$ and $p+p$ collisions are analyzed with Tsallis distributions in different approaches. The information about the freeze-out surface in terms of freeze-out volume, temperature, chemical potential, and radial flow velocity for $\pi^{+}, K^{+}$, and $p$ and their antiparticles is obtained. Furthermore, these parameters are studied as a function of the mass of the particles. A mass dependent differential freeze-out is observed which does not seem to distinguish between particles and their antiparticles. Furthermore, a mass-hierarchy in the radial flow is observed, meaning heavier particles suffer lower radial flow. Tsallis distribution function at finite chemical potential is used to study the mass dependence of chemical potential. The peripheral heavy-ion and proton-proton collisions at the same energies seem to be equivalent in terms of the extracted thermodynamic parameters.
\end{abstract}

\section{Introduction}

High-energy heavy-ion collisions provide a unique opportunity to study the nuclear matter under extreme conditions, that is, at high temperature and/or density. Due to high multiplicities produced in $A+A$ and $p+p$ collisions, the statistical models are more suitable to describe the particle production mechanism. Such a statistical description of transverse momentum $\left(p_{T}\right)$ of final state particles produced in high-energy collisions has been proposed to follow a thermalized Boltzmann type of distribution as given by [1]

$$
E \frac{d^{3} \sigma}{d^{3} p} \simeq C \exp \left(-\frac{p_{T}}{T_{\exp }}\right) .
$$

To account for the high- $p_{T}$ tail, a power-law in $p_{T}$ has been proposed [2-4], which empirically accounts for the possible QCD contributions. Hagedorn proposed a combination of both the aspects, which describes the experimental data over a wide $p_{T}$ range [5] and is given by

$$
E \frac{d^{3} \sigma}{d^{3} p}=C\left(1+\frac{p_{T}}{p_{0}}\right)^{-n} \rightarrow \begin{cases}\exp \left(-\frac{n p_{T}}{p_{0}}\right) & \text { for } p_{T} \longrightarrow 0, \\ \left(\frac{p_{0}}{p_{T}}\right)^{n} & \text { for } p_{T} \longrightarrow \infty,\end{cases}
$$

where $C, p_{0}$, and $n$ are fitting parameters. This becomes a purely exponential function for small $p_{T}$ and a purely power-law function for large $p_{T}$ values. A finite degree of deviation from the equilibrium statistical description of identified particle $p_{T}$ spectra has already been observed by experiments at RHIC $[6,7]$ and LHC [8-11]. Contrary to a thermalized system, where $\left\langle p_{T}\right\rangle$ is associated with the temperature of the hadronizing matter, one fails to make such a connection in case of systems which are far from thermal equilibrium. In the latter systems, the temperature fluctuates either event by event or within the same event [12]. 
This creates room for possible description of the $p_{T}$ spectra in high-energy hadronic and nuclear collisions, using the nonextensive Tsallis statistics [13-15]. A thermodynamically consistent nonextensive distribution function is given by [16]

$$
f\left(m_{T}\right)=C_{q}\left[1+(q-1) \frac{m_{T}}{T}\right]^{-1 /(q-1)} .
$$

Here, $m_{T}$ is the transverse mass and $q$ is called the nonextensive parameter, a measure of degree of deviation from equilibrium. Equations (2) and (3) are related through the following transformations for large values of $p_{T}$ :

$$
\begin{gathered}
n=\frac{1}{q-1}, \\
p_{0}=\frac{T}{q-1} .
\end{gathered}
$$

In the limit $q \rightarrow 1$, one recovers the standard BoltzmannGibbs distribution (see (1)) from the Tsallis distribution (see (6)). Here the effective kinetic freeze-out temperature $\left(T_{\exp }\right)$ as obtained from the inverse slope of the $p_{T}$-spectra using (1) is related to the Tsallis temperature $(T)$ by

$$
T_{\exp } \simeq a+b \cdot T
$$

where $a=0.31-0.654 q+0.354 q^{2}$ and $b=27.35-55 q+29.07 q^{2}$ and they are obtained numerically for distributions with same mean transverse momentum, $\left\langle p_{T}\right\rangle$, as discussed in [17] for $p+p$ collisions in the low $p_{T}\left(0.15<p_{T}<0.6 \mathrm{GeV} / \mathrm{c}\right)$ regime.

Tsallis statistics is used widely to explain the particle spectra in high-energy collisions [12, 22-27] starting from elementary $e^{+}+e^{-}$, hadronic, and heavy-ion collisions $[6,28-$ 44]. The produced particles from the collisions carry the information about collision dynamics and the subsequent space-time evolution till the occurrence of the final freezeout. The evolution of the partonic system created in highenergy experiments is generally believed to be best described by hydrodynamics of an almost ideal fluid [45]. This approach gives a fair description of data on the transverse momentum spectra of hadrons, which are treated as one of the important tools to understand the production dynamics of particles in high-energy collisions. The systematic analysis with the help of an appropriate model or approach guides us to understand various thermodynamical as well as hydrodynamical properties of the fireball at different stages of its evolution. The integrated yields of various hadronic species at different center of mass energies are used in the present work. The corresponding freeze-out parameters for each hadronic species at the time of their freeze-out can be obtained from the analysis of their respective transverse momentum distributions. Different forms of the invariant yields using Tsallis distribution are available in the literature [24, 46, 47]. In the present work we have used all of these forms to study the temperature $(T)$, chemical potential $(\mu)$, radial flow $(\beta)$, volume $(V)$, and nonextensive parameter $(q)$. It should be mentioned here that the parameter $V$ is not necessarily related to the volume one obtains from HBT like experimental measurements. Further, we study the mass dependence of these parameters, which are obtained by analyzing invariant transverse momentum spectra. For the present analysis, we have used the data of $p+p$ and $A+A$ collisions of different experiments at RHIC and LHC. We observe a clear mass dependence of the above parameters, and the behaviour is found to be consistent from most peripheral $A+A$ collisions to $p+p$ collisions. The obtained thermodynamic parameters in $p+p$ collisions are similar to those extracted for most peripheral $A+A$ collisions at the same center of mass energies. This indicates a thermodynamical similarity between both the systems at a given collision energy.

In the heavy-ion collision, the interaction volume of fireball decreases from most central to most peripheral collisions. So the number of participant nucleons also decreases from most central collisions to most peripheral collisions depending on the interaction volume. The system having more participants will quickly reach the equilibrium because of large number of binary collisions by rescattering of partons/hadrons as can be the case in central $A+$ $A$ collisions. But in case of peripheral collisions due to smaller number of participants the system will be away from equilibrium for a while as compared to central collisions. Such a nonequilibrium system is better described by Tsallis nonextensive statistics, giving information about the various thermodynamic parameters of the system.

The paper is organized as follows. In Section 2, we present three forms of Tsallis distribution functions. Firstly, we discuss invariant yields with and without chemical potential in the Tsallis function. Then we show the Tsallis form of invariant yields with radial flow which is introduced analytically in one of our recent works [24]. In Section 3, results and discussions are made. Lastly, we conclude our findings in Section 4.

\section{Nonextensivity and Transverse Momentum Spectra}

In Sections 2 and 3, we discuss the transverse momentum spectra of identified particles $\left(\pi^{+}, K^{+}\right.$, and $p$ and their antiparticles) produced in RHIC and LHC experiments using different forms of invariant yields using Tsallis nonextensive statistics.

2.1. Nonextensive Statistics without Radial Flow. The Tsallis distribution function at mid-rapidity, with finite chemical potential and without radial flow [46], is given by

$$
\begin{aligned}
& \left.\frac{1}{p_{T}} \frac{d^{2} N}{d p_{T} d y}\right|_{y=0} \\
& \quad=\frac{g V m_{T}}{(2 \pi)^{2}}\left[1+(q-1) \frac{m_{T}-\mu}{T}\right]^{-q /(q-1)},
\end{aligned}
$$

where $m_{T}$ is the transverse mass of a particle given by $\sqrt{p_{T}^{2}+m^{2}}, g$ is the degeneracy, and $\mu$ is the chemical potential of the system. In view of higher center of mass energies, where 
$\mu \simeq 0$, the transverse momentum distribution function [47] becomes

$$
\left.\frac{1}{p_{T}} \frac{d^{2} N}{d p_{T} d y}\right|_{y=0}=\frac{g V m_{T}}{(2 \pi)^{2}}\left[1+(q-1) \frac{m_{T}}{T}\right]^{-q /(q-1)} .
$$

2.2. Nonextensive Statistics with Radial Flow. The value of the nonextensive parameter $q$ for high-energy collisions is $1 \leq q \leq 1.2$ [48]. To study the order of deviation of the $p_{T}$-spectra from an equilibrium Boltzmann distribution, the Tsallis distribution function has been expanded in a Taylor series in view of $(q-1)$ being very small, after successful inclusion of radial flow in a relativistic scenario. The details of the method are described in [24]. The functional form of the distribution up to first order in $(q-1)$ is given by

$$
\begin{aligned}
\frac{1}{2 \pi p_{T}} & \frac{d N}{d p_{T} d y} \\
& =\frac{g V}{(2 \pi)^{3}}\left\{2 T\left[r I_{0}(s) K_{1}(r)-s I_{1}(s) K_{0}(r)\right]\right. \\
& -(q-1) \operatorname{Tr}^{2} I_{0}(s)\left[K_{0}(r)+K_{2}(r)\right] \\
+ & 4(q-1) \operatorname{Trs}_{1}(s) K_{1}(r) \\
- & (q-1) \operatorname{Ts}^{2} K_{0}(r)\left[I_{0}(s)+I_{2}(s)\right] \\
+ & \frac{(q-1)}{4} \operatorname{Tr}^{3} I_{0}(s)\left[K_{3}(r)+3 K_{1}(r)\right] \\
- & \frac{3(q-1)}{2} \operatorname{Tr}^{2} s\left[K_{2}(r)+K_{0}(r)\right] I_{1}(s) \\
+ & \frac{3(q-1)}{2} \operatorname{Ts}^{2} r\left[I_{0}(s)+I_{2}(s)\right] K_{1}(r) \\
& \left.-\frac{(q-1)}{4} \operatorname{Ts}^{3}\left[I_{3}(s)+3 I_{1}(s)\right] K_{0}(r)\right\},
\end{aligned}
$$

where $r \equiv \gamma m_{T} / T, s \equiv \gamma \beta p_{T} / T . I_{n}(s)$ and $K_{n}(r)$ are the modified Bessel functions of the first and second kind. There are four parameters involved, namely, $V, T, \beta$, and $q$, where $V$ is the volume, $T$ is the Tsallis temperature, $\beta$ is the radial flow velocity, and $q$ is the Tsallis nonextensive parameter.

We use (8) to fit the particle spectra of identified particles in heavy-ion collisions to study the radial flow parameter.

\section{Results and Discussion}

It is expected that the number of binary collisions in a system with smaller number of participant nucleons is quite low. Hence, the probability of mutual interaction (resulting in momentum transfer) between the system quanta partons or hadrons becomes less for the systems with small value of participating nucleons. This makes the system stay away from a possible thermal equilibrium. On the other hand, an appreciable increment in the number of binary collisions is observed in a system possessing a large number of participant nucleons and consequently the system can reach quickly its

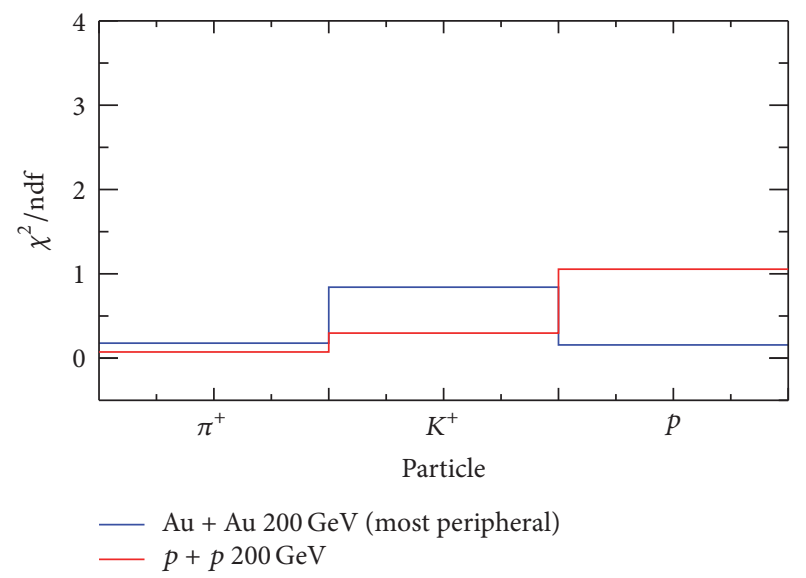

FIGURE 1: $\chi^{2} /$ ndf values are shown for most peripheral $\mathrm{Au}+\mathrm{Au}$ and $p+p$ collisions at $\sqrt{s_{\mathrm{NN}}}=200 \mathrm{GeV}$ using (7) as a fit function.

thermal equilibrium or be in the close vicinity of it. It has been shown in [49] that the nonextensivity parameter $(q)$ is close to 1 in central $\mathrm{Au}+\mathrm{Au}$ collisions and increases towards peripheral collisions. A higher value of $q$ reflects that the system is away from thermal equilibrium. These results suggest that the degree of nonequilibrium is higher in peripheral collisions. Such systems are best described by Tsallis distributions, for example, the most peripheral $A+A$ and $p+p$ systems. We perform the fitting of $p_{T}$ spectra for $p_{\mathrm{T}}<3 \mathrm{GeV} / \mathrm{c}$ around mid-rapidity at $\sqrt{s_{\mathrm{NN}}}=200 \mathrm{GeV}$ and $\sqrt{s_{\mathrm{NN}}}=2.76 \mathrm{TeV}$. Keeping all the parameters free, we try to fit the spectra with (6), (7), and (8) independently for different particles like $\pi^{+}, K^{+}$, and $p$ and their antiparticle for $\mathrm{Pb}+$ $\mathrm{Pb}$ and $\mathrm{Au}+\mathrm{Au}$ most peripheral collisions using TMinuit class available in the ROOT library [50] to get a convergent solution. The same procedure is followed for $p+p$ collisions as well.

We have discussed the present work in three sections. Now, (7) is used to analyze invariant $p_{T}$ spectra of identified particles $\left(\pi^{+}, K^{+}\right.$, and $p$ and their antiparticle) for most peripheral $\mathrm{Au}+\mathrm{Au}$ collisions at $\sqrt{s_{\mathrm{NN}}}=200 \mathrm{GeV}$ and most peripheral $\mathrm{Pb}+\mathrm{Pb}$ collisions at $\sqrt{s_{\mathrm{NN}}}=2.76 \mathrm{TeV}$ along with $p+p$ collisions at both energies. The fitting of $p_{T}$ spectra of identified particles for $\mathrm{Au}+\mathrm{Au}$ collisions at $200 \mathrm{GeV}$ and $\mathrm{Pb}+\mathrm{Pb}$ collisions at $2.76 \mathrm{TeV}$ are shown in Figures 11 and 12 for most peripheral collisions. Also, Figures 13 and 14 show the fitting of invariant spectra to $p+p$ collisions at $200 \mathrm{GeV}$ and $2.76 \mathrm{TeV}$, respectively, for identified particles. The fitting is performed up to the maximum $p_{T}$ of $3 \mathrm{GeV} / \mathrm{c}$ for $A+A$ collisions and $2.5 \mathrm{GeV} / \mathrm{c}$ for $p+p$ collisions although the $K^{+} p_{T}$ spectra in $\mathrm{Au}+\mathrm{Au}$ collision are fitted only up to $2.0 \mathrm{GeV} / \mathrm{c}$ due to unavailability of data [18]. The fittings of Tsallis function with $\mu=0$ seem to be better for peripheral heavy-ion collisions. $\chi^{2} / \mathrm{ndf}$ obtained by minimization in TMinuit are shown in Figures 1 and 2.

A systematic study of the extracted parameters like volume $(V)$, temperature $(T)$, and Tsallis nonextensive parameter $(q)$ is made with identified particle masses. The values of extracted parameters are summarized in Table 1 . We have 


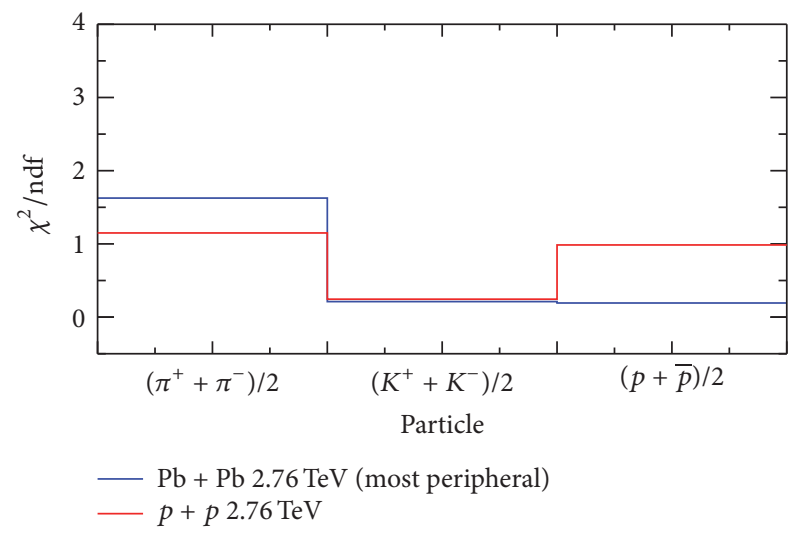

FIgURE 2: $\chi^{2} /$ ndf values are shown for most peripheral $\mathrm{Pb}+\mathrm{Pb}$ and $p+p$ collisions at $\sqrt{s_{\mathrm{NN}}}=2.76 \mathrm{TeV}$ using (7) as a fit function.

TABLE 1: $\chi^{2} /$ ndf and different extracted parameters from Tsallis distribution fit (see (7)) to the $p_{T}$ spectra of (a) $\pi^{+}, K^{+}$, and $p$ for most peripheral $\mathrm{Au}+\mathrm{Au}$ collisions and $p+p$ collisions at $\sqrt{s_{\mathrm{NN}}}$ $=200 \mathrm{GeV}$; (b) $\left(\pi^{+}+\pi^{-}\right) / 2,\left(K^{+}+K^{-}\right) / 2$, and $(p+\bar{p}) / 2$ for most peripheral $\mathrm{Pb}+\mathrm{Pb}$ collisions and $\left(\pi^{+}+\pi^{-}\right) / 2,\left(K^{+}+K^{-}\right) / 2$ and $(p+\bar{p}) / 2$ for $p+p$ collisions at $\sqrt{s_{\mathrm{NN}}}=2.76 \mathrm{TeV}$.

(a)

\begin{tabular}{lcccc}
\hline \multicolumn{5}{c}{$\mathrm{Au}+\mathrm{Au} 200 \mathrm{GeV}$ (peripheral) } \\
Particle & $\chi^{2} / \mathrm{ndf}$ & $V(\mathrm{fm})^{3}$ & $T(\mathrm{GeV})$ & $q$ \\
\hline$\pi^{+}$ & 0.178 & $2242.35 \pm 18.23$ & $0.093 \pm 0.003$ & $1.113 \pm 0.004$ \\
$K^{+}$ & 0.842 & $223.358 \pm 0.010$ & $0.085 \pm 0.011$ & $1.120 \pm 0.002$ \\
$p$ & 0.157 & $45.62 \pm 2.45$ & $0.205 \pm 0.012$ & $1.025 \pm 0.029$ \\
\hline \multicolumn{5}{c}{$p+p 200 \mathrm{GeV}$} \\
\hline$\pi^{+}$ & 0.073 & $5293.1 \pm 23.3$ & $0.088 \pm 0.004$ & $1.103 \pm 0.005$ \\
$K^{+}$ & 0.297 & $2443.7 \pm 33.7$ & $0.066 \pm 0.002$ & $1.161 \pm 0.009$ \\
$p$ & 1.055 & $360.5 \pm 9.6$ & $0.113 \pm 0.004$ & $1.070 \pm 0.000$ \\
\hline
\end{tabular}

(b)

\begin{tabular}{|c|c|c|c|c|}
\hline \multicolumn{5}{|c|}{$\mathrm{Pb}+\mathrm{Pb} 2.76 \mathrm{TeV}$ (peripheral) } \\
\hline Particle & $\chi^{2} / \mathrm{ndf}$ & $V(\mathrm{fm})^{3}$ & $T(\mathrm{GeV})$ & $q$ \\
\hline$\frac{\pi^{+}+\pi^{-}}{2}$ & 1.626 & $6134.30 \pm 12.61$ & $0.082 \pm 0.001$ & $1.156 \pm 0.002$ \\
\hline$\frac{K^{+}+K^{-}}{2}$ & 0.211 & $629.69 \pm 14.80$ & $0.133 \pm 0.003$ & $1.120 \pm 0.004$ \\
\hline$\frac{p+\bar{p}}{2}$ & 0.193 & $39.623 \pm 1.052$ & $0.238 \pm 0.012$ & $1.052 \pm 0.009$ \\
\hline \multicolumn{5}{|c|}{$p+p 2.76 \mathrm{TeV}$} \\
\hline$\frac{\pi^{+}+\pi^{-}}{2}$ & 0.985 & $1102.46 \pm 6.82$ & $0.070 \pm 0.001$ & $1.163 \pm 0.001$ \\
\hline$\frac{K^{+}+K^{-}}{2}$ & 0.094 & $127.13 \pm 1.33$ & $0.096 \pm 0.005$ & $1.144 \pm 0.005$ \\
\hline$\frac{p+\bar{p}}{2}$ & 0.006 & $291.39 \pm 10.62$ & $0.069 \pm 0.003$ & $1.133 \pm 0.002$ \\
\hline
\end{tabular}

calculated the radius of the fireball at freeze-out and plotted it as a function of the mass of the identified particles. Figures 3 and 4 show the variation of common mass dependent parameters at $\sqrt{s_{\mathrm{NN}}}=200 \mathrm{GeV}$ and $\sqrt{s_{\mathrm{NN}}}=2.76 \mathrm{TeV}$. We have then compared the parameters obtained for peripheral heavy-ion collisions with that of $p+p$ collisions at the same center of mass energies to understand the thermodynamic properties of the produced systems.

It is found that the volume parameter decreases with increase in particle mass. It indicates that different particles have different freeze-out surfaces depending on their mass. This is observed for both $p+p$ and $A+A$ collisions and thus indicates a mass dependent differential freeze-out scenario in high-energy collisions. In view of the above freeze-out picture, taking $T$ as the freeze-out temperature, we observe from Figures 3 and 4 that the freeze-out temperature slightly increases with particle mass. This observation goes in line with the earlier findings of [51], which uses a slightly different approach. It clearly indicates that the heavier particles have higher freeze-out temperature as compared to lighter particles. This observation goes in line with an intuitive expectation of mass dependent particle freeze-out or differential freeze-out. In literature, one finds various freeze-out scenarios like single freeze-out [26], strange and nonstrange particles having two different freeze-outs $[52,53]$, and so forth. However, our findings from the analysis of highenergy particle spectra with nonextensive statistics reveal a consistent differential freeze-out scenario with particles and their counter parts, the antiparticles freezing out at the same time (characterized by freeze-out temperature, freezeout volume) from the produced fireballs. The nonextensive measure, that is, the $q$-parameter obtained from our studies, is consistent with its value, $1<q<1.2$, for both $p+p$ and $A+A$ collisions. This goes in line with the expectations of the $q$-values in high-energy collisions [48].

Also, Figures 3 and 4 indicate a similar mass ordering for $p+p$ systems, which indicates that the systems formed in most peripheral $A+A$ collisions and $p+p$ collisions are of similar thermodynamic nature. However, it should be noted that the obtained parameters seem to be unphysical; that is, volume or the radius in $p+p$ collisions seems to be higher than the peripheral $A+A$ collisions at the same energy. This problem is observed to be circumvented, when one adds the radial flow to the distribution. This is seen in the subsequent discussions and in Table 3.

Secondly, we perform the fitting of invariant $p_{T}$ spectra of identified particles using (6) which contains an additional parameter as chemical potential $(\mu)$. The same fitting procedure is used to fit $\mathrm{Au}+\mathrm{Au}$ most peripheral collisions at $\sqrt{s_{\mathrm{NN}}}=200 \mathrm{GeV}$ for identified particles as well as their antiparticles. The Tsallis form of invariant yields including $\mu$ is used in Figure 15. It fits up to $p_{T} \sim 3 \mathrm{GeV} / \mathrm{c}$ for most peripheral collisions. The goodness of fit can be seen from $\chi^{2} /$ ndf values shown in Figure 5.

The mass dependence of extracted parameters is shown in Figure 6. Also, the values of the parameters are tabulated in Table 2. In Figure 6, it is shown that all the parameters follow the same mass dependent trend as is discussed in the previous cases. Besides, an additional parameter, chemical potential $(\mu)$, is shown.

It is to be mentioned here that the chemical potential is extracted from the invariant yields at the thermal freeze-out, 
TABLe 2: $\chi^{2} /$ ndf and different extracted parameters from Tsallis distribution fit (see (6)) to the $p_{T}$ spectra of $\pi^{ \pm}, K^{ \pm}, p$, and $\bar{p}$ for most peripheral $\mathrm{Au}+\mathrm{Au}$ collisions at $\sqrt{s_{\mathrm{NN}}}=200 \mathrm{GeV}$.

\begin{tabular}{lccccc}
\hline & & \multicolumn{2}{c}{$\mathrm{Au}+\mathrm{Au} 200 \mathrm{GeV}$ (peripheral) } & & \\
Particle & $\chi^{2} / \mathrm{ndf}$ & $V(\mathrm{fm})^{3}$ & $T(\mathrm{GeV})$ & $1.113 \pm 0.004$ & $0.0050 \pm 0.0003$ \\
\hline$\pi^{+}$ & 0.191 & $2125.93 \pm 56.06$ & $0.094 \pm 0.004$ & $1.110 \pm 0.004$ & $0.0080 \pm 0.0003$ \\
$\pi^{-}$ & 0.325 & $1895.78 \pm 275.34$ & $0.097 \pm 0.004$ & $1.128 \pm 0.025$ & $0.086 \pm 0.009$ \\
$K^{+}$ & 1.415 & $63.64 \pm 12.60$ & $0.089 \pm 0.021$ & $1.126 \pm 0.023$ & $0.085 \pm 0.003$ \\
$K^{-}$ & 1.309 & $113.82 \pm 68.83$ & $0.090 \pm 0.012$ & $1.026 \pm 0.024$ & $0.310 \pm 0.190$ \\
$p$ & 0.170 & $10.01 \pm 3.71$ & $0.213 \pm 0.029$ & $1.051 \pm 0.014$ & $0.437 \pm 0.103$ \\
$\bar{p}$ & 0.111 & $11.62 \pm 3.48$ & $0.183 \pm 0.015$ & & \\
\hline
\end{tabular}
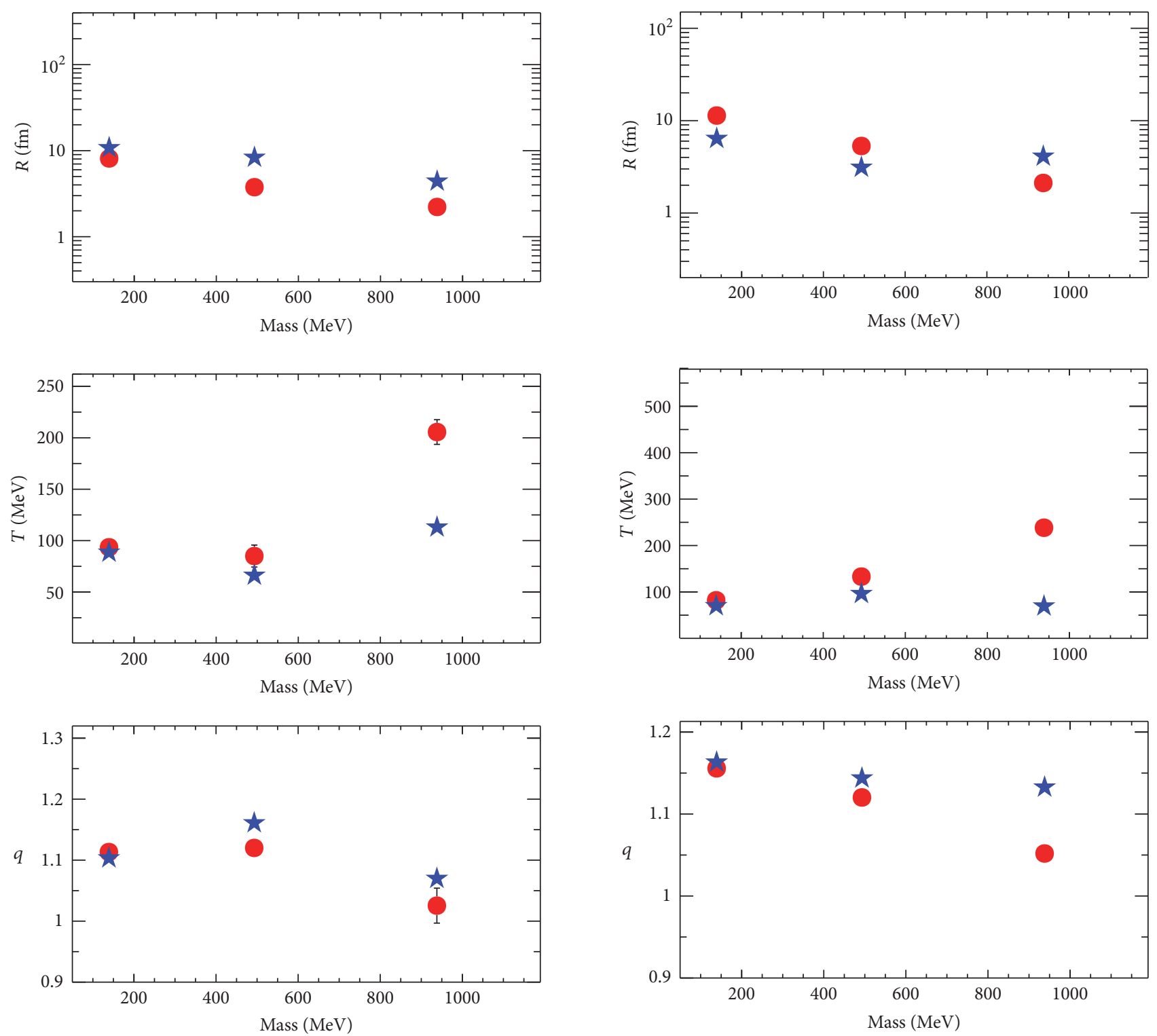

$\mathrm{Au}+\mathrm{Au} 200 \mathrm{GeV}$ (most peripheral) $\star$ \ $p+p 200 \mathrm{GeV}$

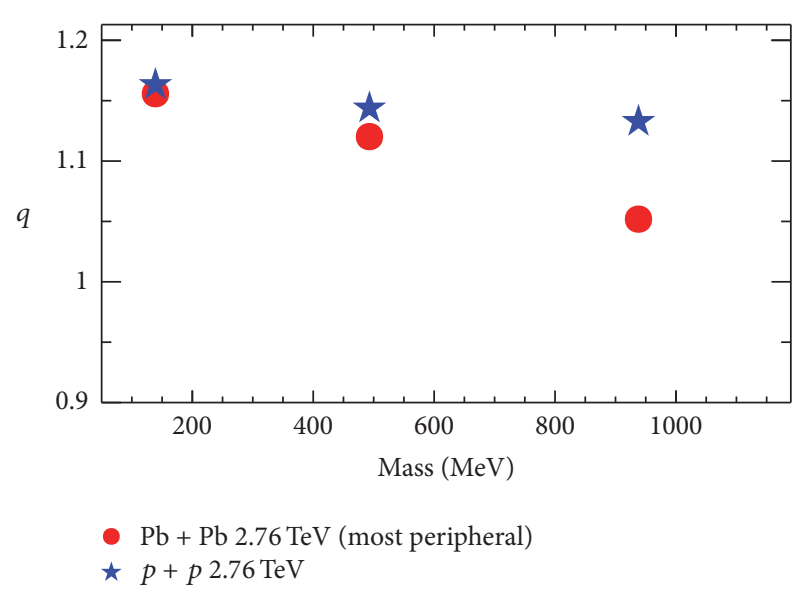

FIGURE 3: Mass dependence of the thermodynamic parameters using (7) for most peripheral $\mathrm{Au}+\mathrm{Au}$ and $p+p$ collisions at $\sqrt{s_{\mathrm{NN}}}$ $=200 \mathrm{GeV}$.

FIgURE 4: Mass dependence of thermodynamic parameters using (7) for most peripheral $\mathrm{Pb}+\mathrm{Pb}$ and $p+p$ collisions at $\sqrt{s_{\mathrm{NN}}}=$ $2.76 \mathrm{TeV}$. 


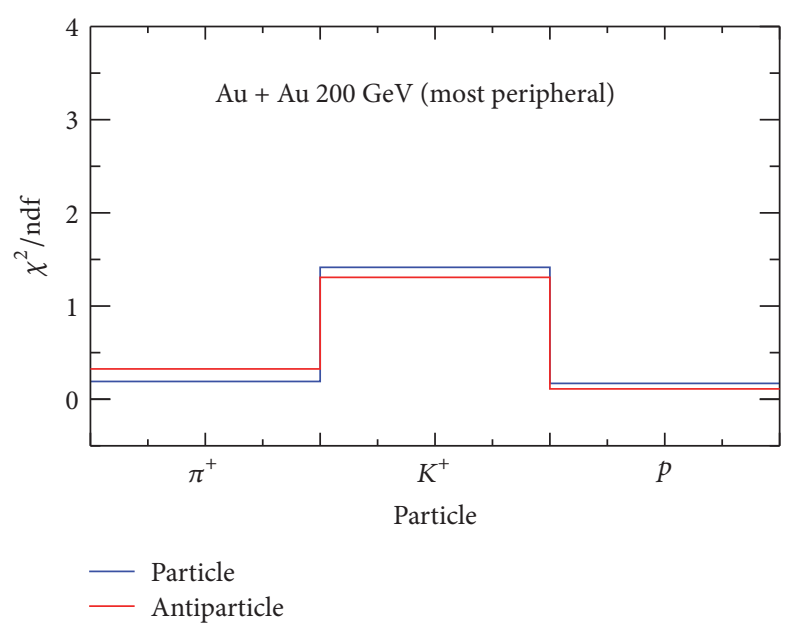

Figure 5: $\chi^{2} / \mathrm{ndf}$ values are shown for most peripheral $\mathrm{Au}+\mathrm{Au}$ collisions at $\sqrt{s_{\mathrm{NN}}}=200 \mathrm{GeV}$ using (6) as a fit function.
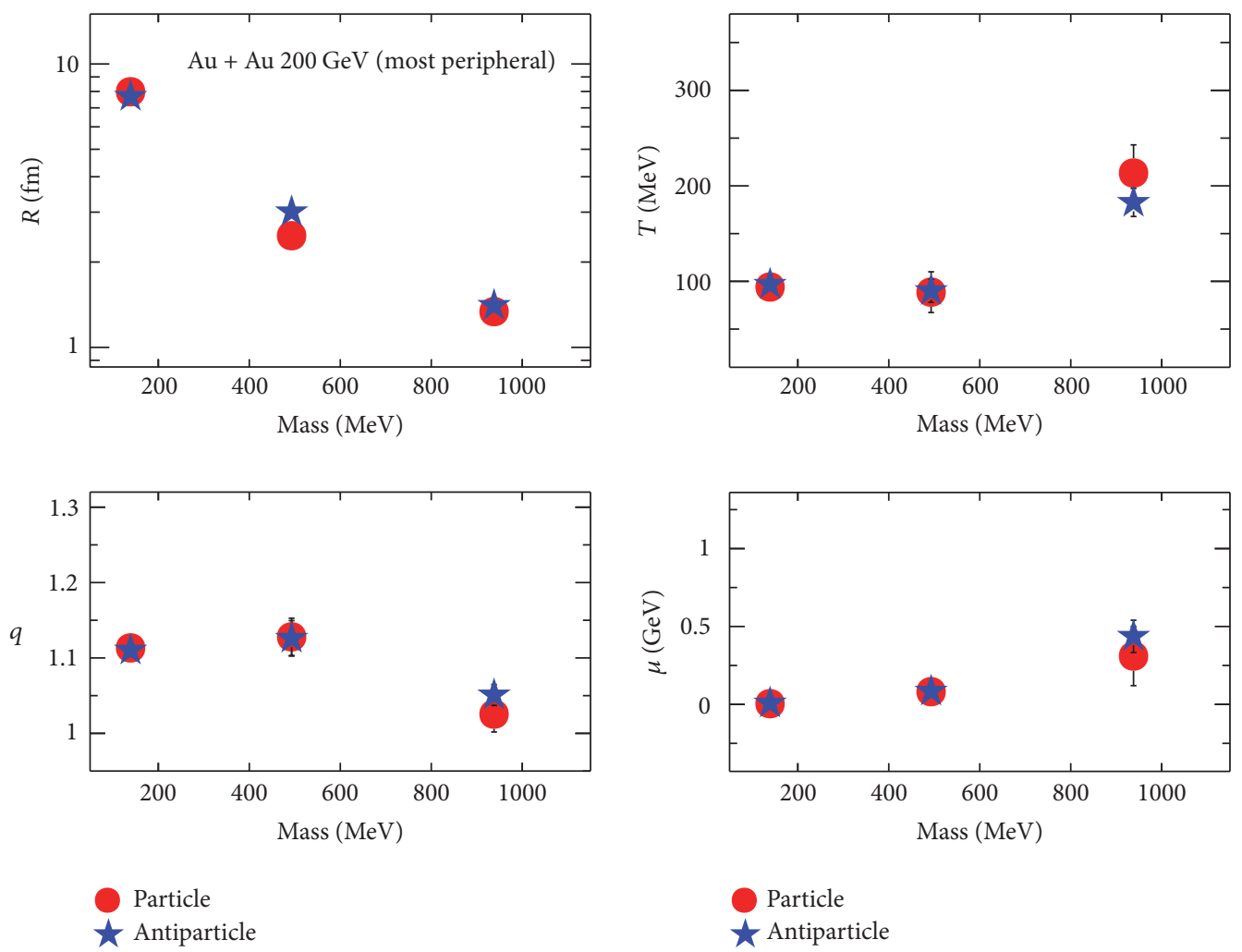

FIGURE 6: Mass dependence of extracted thermodynamic parameters using (6) for different particles and their corresponding antiparticles for most peripheral $\mathrm{Au}+\mathrm{Au}$ collisions at $\sqrt{s_{\mathrm{NN}}}=200 \mathrm{GeV}$.

where all the elastic processes cease. Hence the obtained chemical potential here is called thermal chemical potential. At this stage one can introduce chemical potentials for all particle species $\left(\mu_{i}\right)$. Following [54], the relationship between chemical potential at kinetic freeze-out and chemical potential at chemical freeze-out is given by

$$
\mu_{\mathrm{th}}=\mu_{\mathrm{ch}} \frac{T_{\mathrm{th}}}{T_{\mathrm{ch}}}+m\left(1-\frac{T_{\mathrm{th}}}{T_{\mathrm{ch}}}\right)
$$

where $\left(T_{\mathrm{ch}}, T_{\mathrm{th}}\right)$ and $\left(\mu_{\mathrm{ch}}, \mu_{\mathrm{th}}\right)$ are temperature and chemical potential at chemical and thermal freeze-out, respectively. Equation (9) clearly shows a mass dependency of thermal chemical potential.

The extracted parameters shown in Figure 6 show a consistent picture for particles and antiparticles in case of most peripheral collisions. It is evident from Figure 6 that the $\mu$-parameter increases with particle mass. It shows that particles and antiparticles have same freeze-out conditions. 


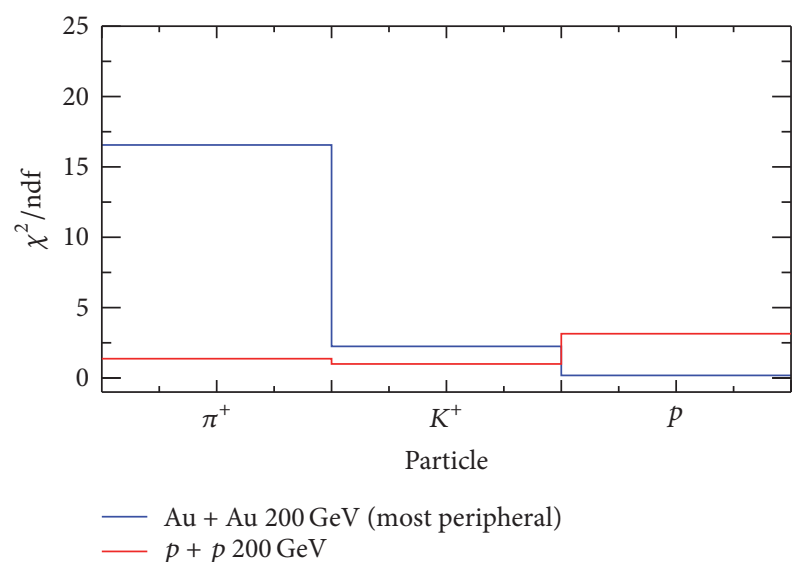

Figure 7: $\chi^{2} /$ ndf values are shown for most peripheral $\mathrm{Au}+\mathrm{Au}$ collision and $p+p$ collisions at $\sqrt{s_{\mathrm{NN}}}=200 \mathrm{GeV}$ using (8) as a fit function.

It is worth mentioning here that the parameters $T$ and $\mu$ extracted from identified particle spectra using (6) are related by [17]

$$
T=T_{0}+(q-1) \mu,
$$

where $T=T_{0}$ for $\mu=0$. Our extracted parameters using (6) (i.e., $T, \mu$, and $q$ ) and (7) (i.e., $T \equiv T_{0}$ ) seem to obey the above relation.

Finally, the invariant $p_{T}$ spectra of identified particles are fitted with (8) for most peripheral collisions of $\mathrm{Au}+\mathrm{Au}$ at $\sqrt{s_{\mathrm{NN}}}=200 \mathrm{GeV}$ and $\mathrm{Pb}+\mathrm{Pb}$ at $\sqrt{s_{\mathrm{NN}}}=2.76 \mathrm{TeV}$. Besides, the fitting of identified particles for $p+p$ collisions is done for the same energies. The fitting results for $\mathrm{Au}+\mathrm{Au}$ collisions at $\sqrt{s_{\mathrm{NN}}}=200 \mathrm{GeV}$ and the results for $\mathrm{Pb}+\mathrm{Pb}$ collisions at $\sqrt{s_{\mathrm{NN}}}$ $=2.76 \mathrm{TeV}$ are shown in Figures 16 and 17, respectively. Also, the fitting results for $p+p$ collisions at $\sqrt{s_{\mathrm{NN}}}=200 \mathrm{GeV}$ and $\sqrt{s_{\mathrm{NN}}}=2.76 \mathrm{TeV}$ are shown in Figures 18 and 19, respectively.

The fitting is performed up to $p_{T} \sim 3 \mathrm{GeV} / \mathrm{c}$ in $A+A$ collisions and $p_{T} \sim 2.5 \mathrm{GeV} / \mathrm{c}$ in $p+p$ collisions; only a slight deviation at higher $p_{T}$ is observed in pion spectra. The values of various parameters obtained from the fits are provided in Table 3. It is observed from the values of $\chi^{2} / \mathrm{ndf}$ (Table 3) that the performance of the present approach is quite satisfactory, except in case of pion, which can be seen from Figures 7 and 8.

Then we make a mass dependent study of these parameters. Figures 9 and 10 represent the behaviour of the extracted parameters. The parameters like volume, temperature, and $q$ show a similar mass dependent behaviour as that of the parameters obtained from the fitting using (6) and (7). Here, through (8), we have introduced an additional radial flow parameter $(\beta)$. Figures 9 and 10 show the decrease of radial flow with increase of particle mass, which is a signature of hydrodynamic evolution of the system created in high-energy collisions. The change of temperature parameter with the use of different forms of Tsallis distribution could be observed in [55]. In our analysis we have also observed that not only the temperature but also the other parameters are changing with the use of different forms of Tsallis distribution. For

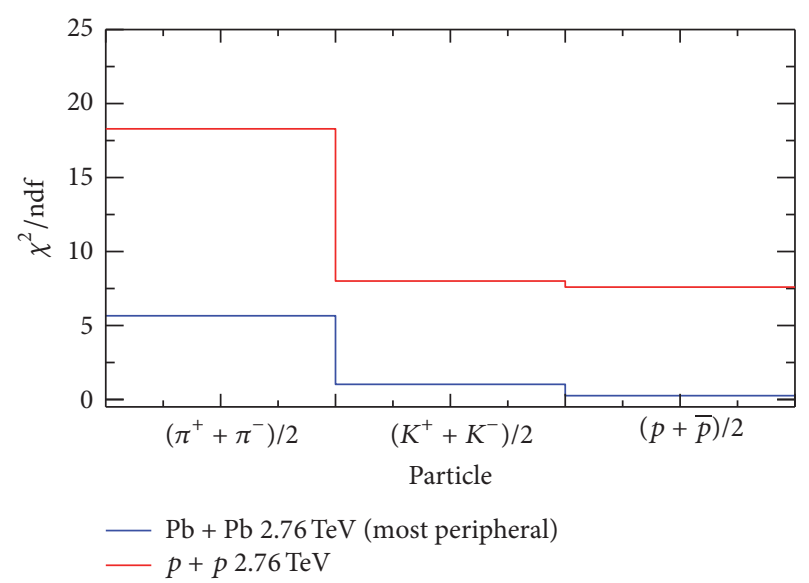

Figure 8: $\chi^{2} /$ ndf values are shown for most peripheral $\mathrm{Pb}+\mathrm{Pb}$ collisions and $p+p$ collisions at $\sqrt{s_{\mathrm{NN}}}=2.76 \mathrm{TeV}$ using (8) as a fit function.

example, it is observed that, with the inclusion of radial flow, the value of the $q$-parameter becomes less, as compared to that observed in the other forms of Tsallis distribution, while describing the $p_{T}$-spectra. This may hint that the nonextensivity is shared by the dynamics of the system [5658].

\section{Conclusion}

We present a systematic study of the parameters extracted from transverse momentum spectra of produced identified particles in $\mathrm{Au}+\mathrm{Au}$ collisions at $\sqrt{s_{\mathrm{NN}}}=200 \mathrm{GeV}$ and $\mathrm{Pb}+\mathrm{Pb}$ collisions at $\sqrt{s_{\mathrm{NN}}}=2.76 \mathrm{TeV}$. Furthermore, the same analysis is done for $p+p$ collisions at both, RHIC and LHC energies. In the present work different forms of invariant yields using Tsallis distributions are used. Parameters obtained from three different forms in most peripheral $\mathrm{Au}+\mathrm{Au}$ collisions at $\sqrt{s_{\mathrm{NN}}}=200 \mathrm{GeV}$ and in most peripheral $\mathrm{Pb}+\mathrm{Pb}$ collisions at $\sqrt{s_{\mathrm{NN}}}=2.76 \mathrm{TeV}$ follow a similar trend. Similar behaviour has been observed in $p+p$ collisions for both the energies. The chemical potential plays an important role in particle production mechanism. We observe that the particles produced at early stage have higher thermal chemical potential as compared to particles produced at the later stage. The radial flow extracted from the particle spectra of peripheral $A+A$ collisions is similar to $p+p$ collisions at the same center of mass energy. In the discussed low- $p_{T}$ region, the radial flow decreases with increasing mass, which goes in line with hydrodynamic description of the evolution of a fireball created in high-energy collisions. Therefore, the present analysis gives a systematic information of hydrodynamical and thermodynamical evolution of the system, while making a direct comparison with hadronic and peripheral nuclear collisions. We observe a mass dependent differential freezeout scenario in high-energy hadronic and nuclear collisions, which is also supported by similar observations by other authors [59]. 

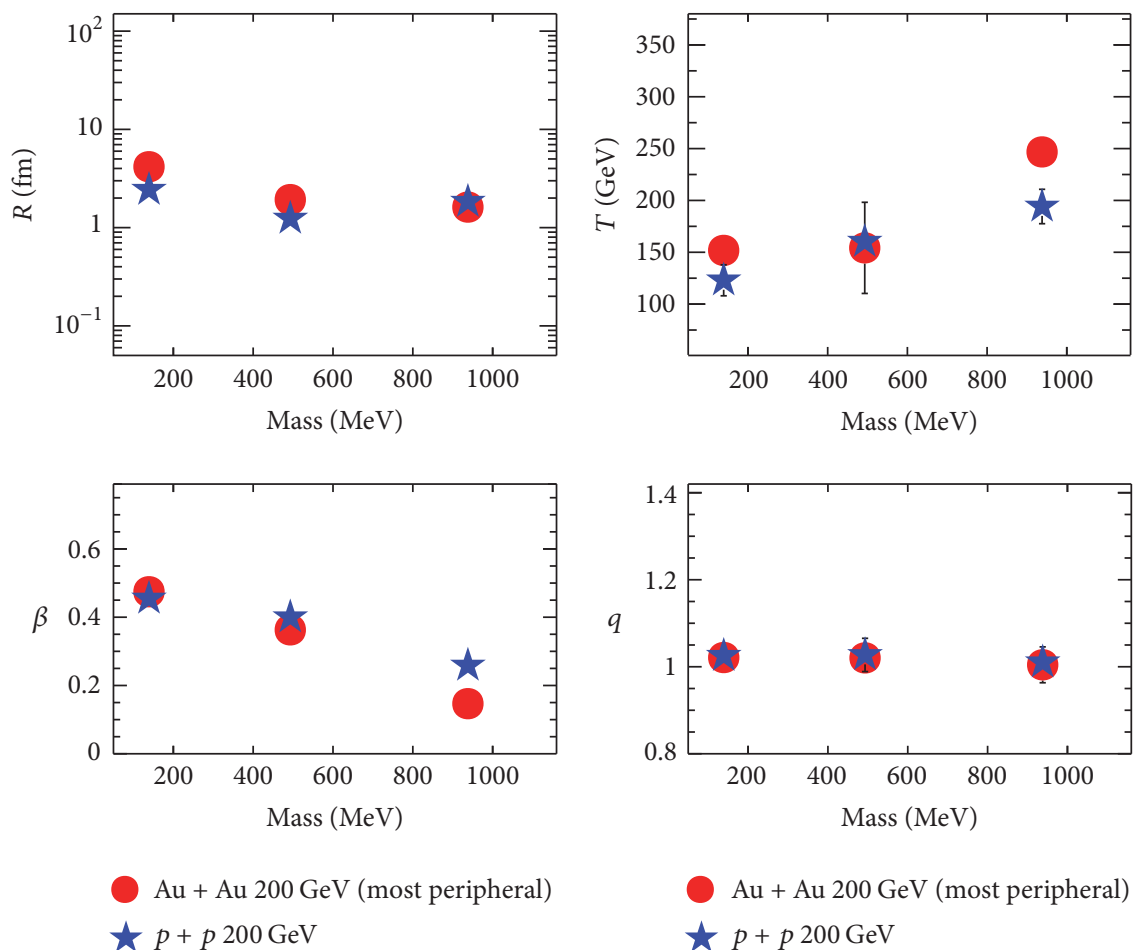

FIGURE 9: Mass dependence of extracted thermodynamic parameters of Tsallis distribution including radial flow using (8) for most peripheral $\mathrm{Au}+\mathrm{Au}$ collisions and $p+p$ collisions at $\sqrt{s_{\mathrm{NN}}}=200 \mathrm{GeV}$.
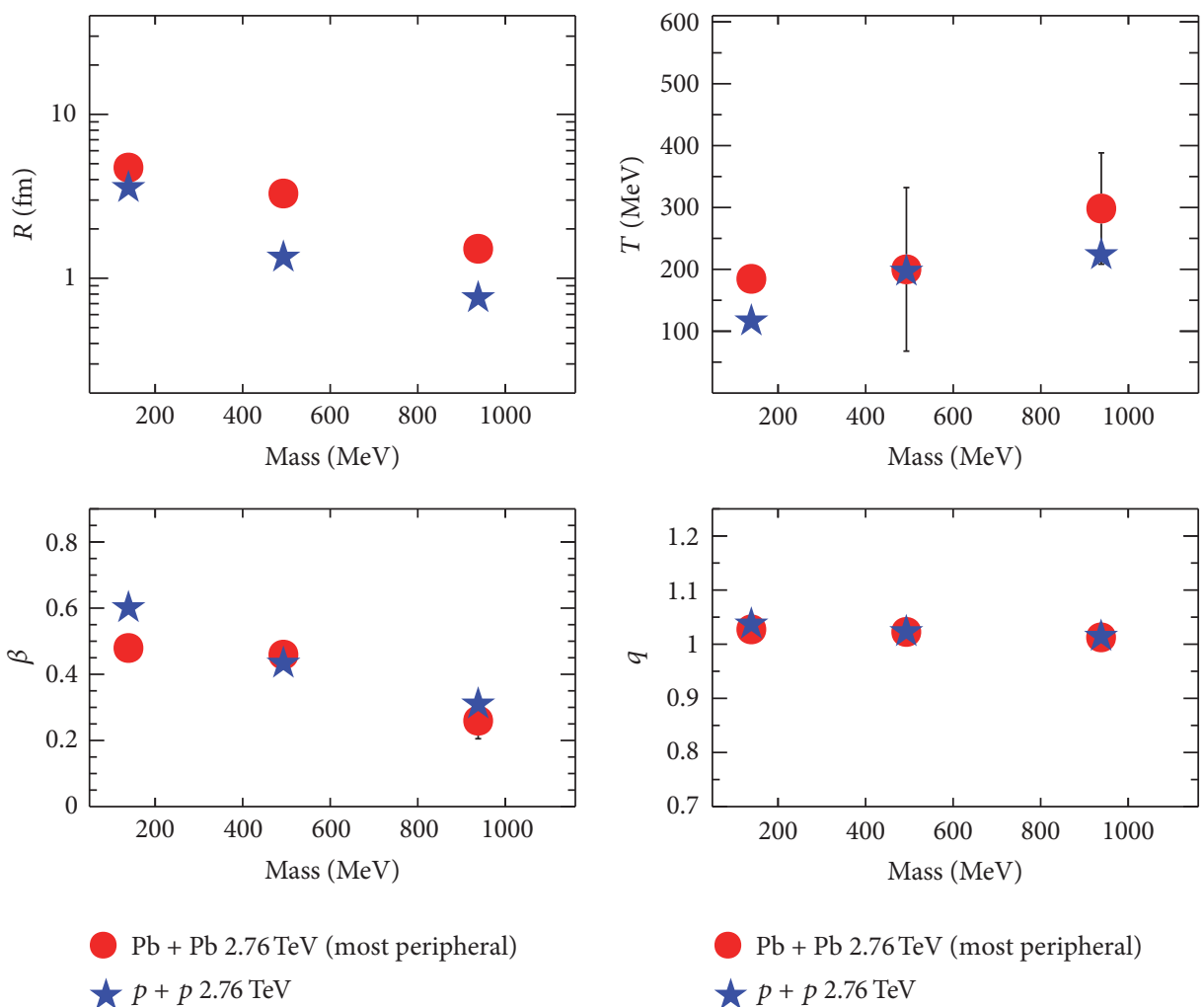

FIGURE 10: Mass dependence of extracted thermodynamic parameters of Tsallis distribution including radial flow using (8) for most peripheral $\mathrm{Pb}+\mathrm{Pb}$ collisions and $p+p$ collisions at $\sqrt{s_{\mathrm{NN}}}=2.76 \mathrm{TeV}$. 
TABLE 3: $\chi^{2} /$ ndf and different extracted parameters from Tsallis distribution fit (see (8)) to the $p_{T}$ spectra of (a) $\pi^{+}, K^{+}$, and $p$ for most peripheral $\mathrm{Au}+\mathrm{Au}$ collisions and $p+p$ collisions at $\sqrt{s_{\mathrm{NN}}}=200 \mathrm{GeV} ;(\mathrm{b})\left(\pi^{+}+\pi^{-}\right) / 2,\left(K^{+}+K^{-}\right) / 2$, and $(p+\bar{p}) / 2$ for most peripheral Pb $+\mathrm{Pb}$ collisions and $\left(\pi^{+}+\pi^{-}\right) / 2,\left(K^{+}+K^{-}\right) / 2$, and $(p+\bar{p}) / 2$ for $p+p$ collisions at $\sqrt{s_{\mathrm{NN}}}=2.76 \mathrm{TeV}$.

(a)

\begin{tabular}{lccccc}
\hline & \multicolumn{2}{c}{$\mathrm{Au}+\mathrm{Au} 200 \mathrm{GeV}$ (peripheral) } \\
Particle & $\chi^{2} / \mathrm{ndf}$ & $V(\mathrm{fm})^{3}$ & $T(\mathrm{GeV})$ & $\beta$ & $q$ \\
\hline$\pi^{+}$ & 16.558 & $302.680 \pm 7.591$ & $0.152 \pm 0.001$ & $0.475 \pm 0.013$ & $1.021 \pm 0.004$ \\
$K^{+}$ & 2.2461 & $30.005 \pm 1.676$ & $0.154 \pm 0.044$ & $0.363 \pm 0.008$ & $1.020 \pm 0.021$ \\
$p$ & 0.1825 & $17.731 \pm 1.952$ & $0.247 \pm 0.000$ & $0.147 \pm 0.018$ & $1.004 \pm 0.041$ \\
\hline & & & $p+p 200 \mathrm{GeV}$ & $1.025 \pm 0.015$ \\
\hline$\pi^{+}$ & 1.370 & $60.260 \pm 0.506$ & $0.123 \pm 0.015$ & $0.455 \pm 0.015$ & $1.027 \pm 0.038$ \\
$K^{+}$ & 0.996 & $7.914 \pm 0.878$ & $0.160 \pm 0.004$ & $0.400 \pm 0.013$ & $1.010 \pm 0.010$ \\
\hline
\end{tabular}

(b)

\begin{tabular}{|c|c|c|c|c|c|}
\hline \multicolumn{6}{|c|}{$\mathrm{Pb}+\mathrm{Pb} 2.76 \mathrm{TeV}$ (peripheral) } \\
\hline Particle & $\chi^{2} / \mathrm{ndf}$ & $V(\mathrm{fm})^{3}$ & $T(\mathrm{GeV})$ & $\beta$ & $q$ \\
\hline$\frac{\pi^{+}+\pi^{-}}{2}$ & 5.657 & $444.714 \pm 7.931$ & $0.185 \pm 0.008$ & $0.480 \pm 0.028$ & $1.027 \pm 0.006$ \\
\hline$\frac{K^{+}+K^{-}}{2}$ & 1.025 & $149.038 \pm 11.465$ & $0.200 \pm 0.133$ & $0.460 \pm 0.021$ & $1.023 \pm 0.010$ \\
\hline$\frac{p+\bar{p}}{2}$ & 0.258 & $14.507 \pm 1.107$ & $0.299 \pm 0.090$ & $0.260 \pm 0.055$ & $1.013 \pm 0.024$ \\
\hline \multicolumn{6}{|c|}{$p+p 2.76 \mathrm{TeV}$} \\
\hline$\frac{\pi^{+}+\pi^{-}}{2}$ & 18.290 & $191.039 \pm 15.890$ & $0.116 \pm 0.003$ & $0.601 \pm 0.013$ & $1.038 \pm 0.004$ \\
\hline$\frac{K^{+}+K^{-}}{2}$ & 8.006 & $10.164 \pm 1.097$ & $0.197 \pm 0.005$ & $0.433 \pm 0.016$ & $1.023 \pm 0.005$ \\
\hline$\frac{p+\bar{p}}{2}$ & 7.596 & $1.839 \pm 0.256$ & $0.224 \pm 0.006$ & $0.310 \pm 0.014$ & $1.014 \pm 0.004$ \\
\hline
\end{tabular}

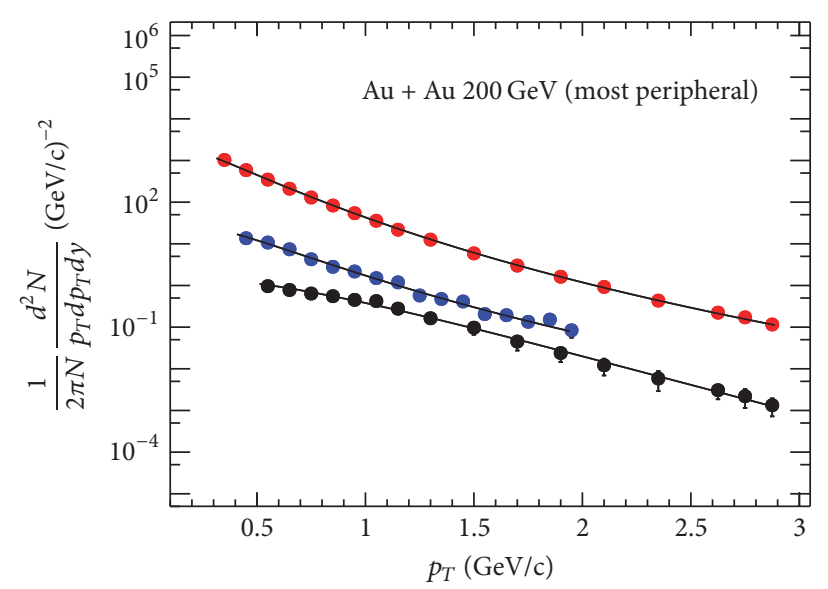

$\pi^{+}(\times 90.5)$

$K^{+}(\times 72.5)$

- $p(\times 3.5)$

Figure 11: Fitting of invariant $p_{T}$ spectra with Tsallis distribution using (7) for $\pi^{+}[18], K^{+}[18]$, and $p$ [18] in most peripheral Au + Au collisions at $\sqrt{s_{\mathrm{NN}}}=200 \mathrm{GeV}$. Symbols represent the data points and line is the fit function. 


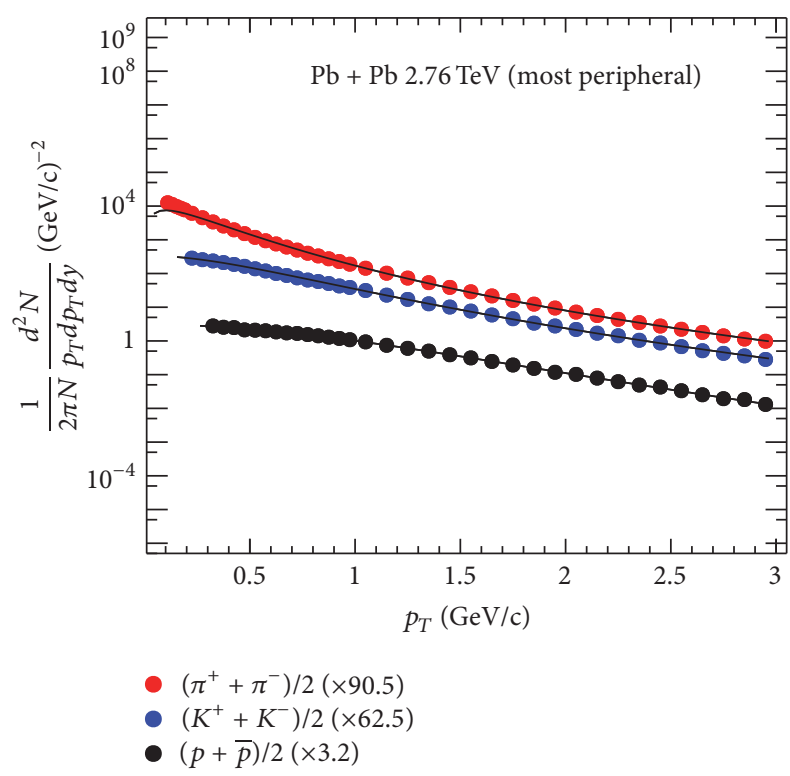

Figure 12: Fitting of invariant $p_{T}$ spectra with Tsallis distribution using $(7)$ for $\left(\pi^{+}+\pi^{-}\right) / 2[19,20],\left(K^{+}+K^{-}\right) / 2[19,20]$, and $(p+\bar{p}) / 2$ $[19,20]$ in most peripheral $\mathrm{Pb}+\mathrm{Pb}$ collisions at $\sqrt{s_{\mathrm{NN}}}=2.76 \mathrm{TeV}$. Symbols represent the data points and line is the fit function.

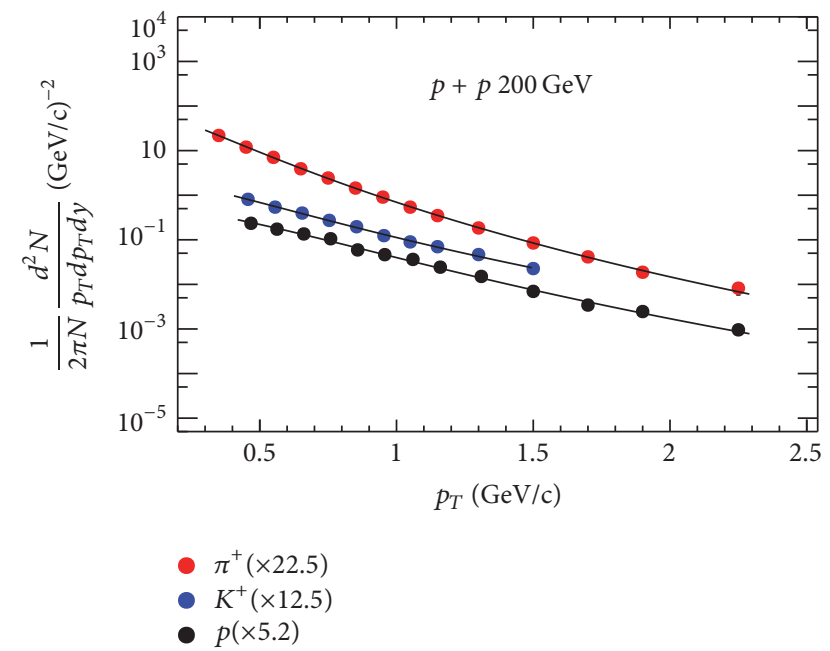

Figure 13: Fitting of invariant $p_{T}$ spectra with Tsallis distribution using (7) for $\pi^{+}$[21], $K^{+}$[21], and $p$ [21] in $p+p$ collisions at $\sqrt{s_{\mathrm{NN}}}=200 \mathrm{GeV}$. Symbols represent the data points and line is the fit function.

\section{Appendix}

See Figures 11-19 and Tables 1-3.

\section{Disclosure}

Prakhar Garg's current affiliation is Department of Physics and Astronomy, Stony Brook University, SUNY, Stony Brook, NY 11794-3800, USA.

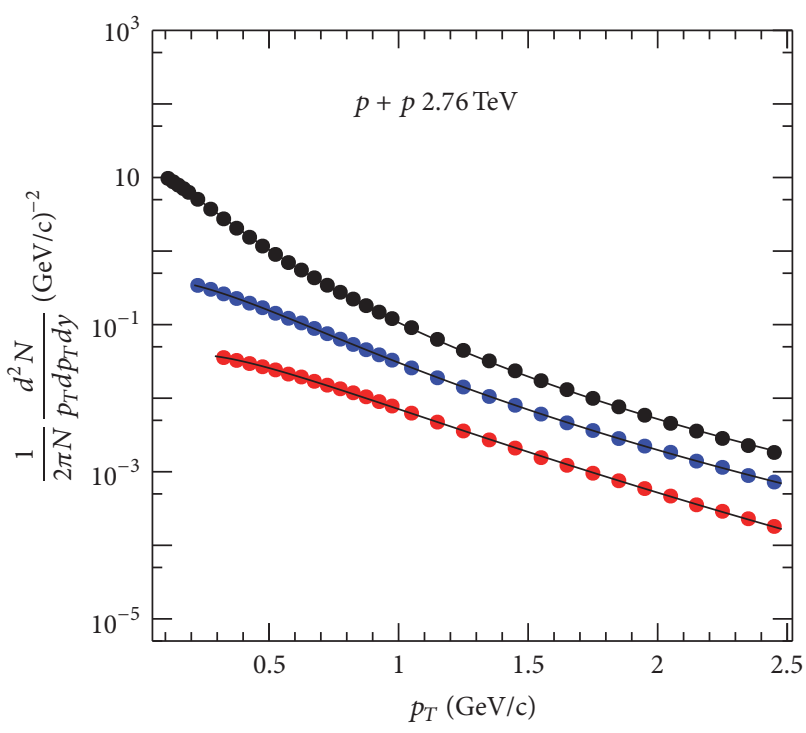

- $\left(\pi^{+}+\pi^{-}\right) / 2$

$\left(K^{+}+K^{-}\right) / 2$

$(p+\bar{p}) / 2(\times 0.4)$

FIGURE 14: Fitting of invariant $p_{T}$ spectra with Tsallis distribution using (7) for $\left(\pi^{+}+\pi^{-}\right) / 2[19,20],\left(K^{+}+K^{-}\right) / 2[19,20]$, and $(p+\bar{p}) / 2$ $[19,20]$ in $p+p$ collisions at $\sqrt{s_{\mathrm{NN}}}=2.76 \mathrm{TeV}$. Symbols represent the data points and line is the fit function.

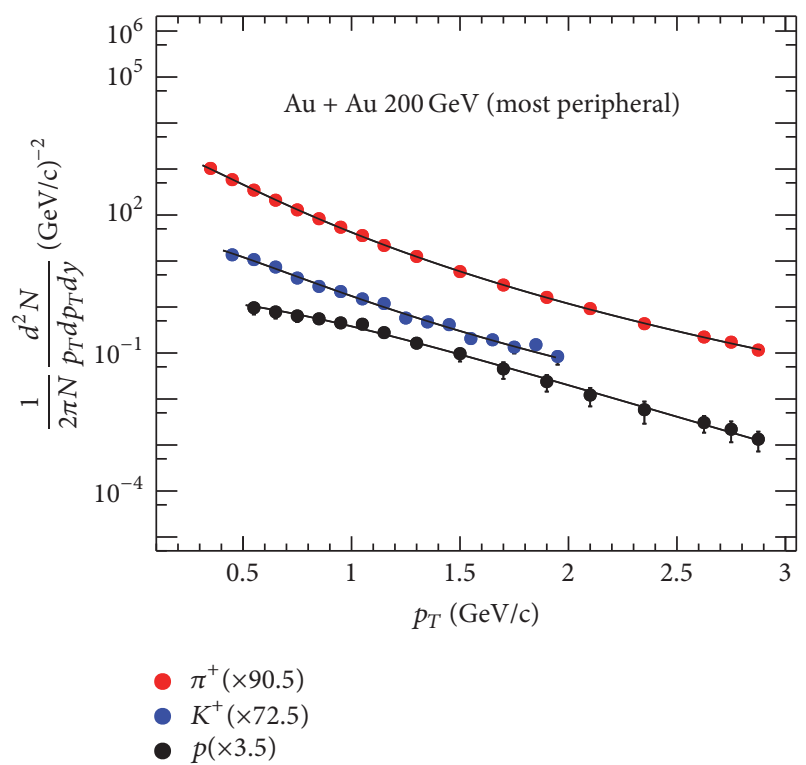

FIGURE 15: Fitting of invariant $p_{T}$ spectra with Tsallis distribution including chemical potential using (6) for $\pi^{+}[18], K^{+}[18]$, and $p$ [18] in most peripheral $\mathrm{Au}+\mathrm{Au}$ collisions at $\sqrt{s_{\mathrm{NN}}}=200 \mathrm{GeV}$. Symbols represent the data points and the line is the fit function.

\section{Competing Interests}

The authors declare that they have no competing interests. 


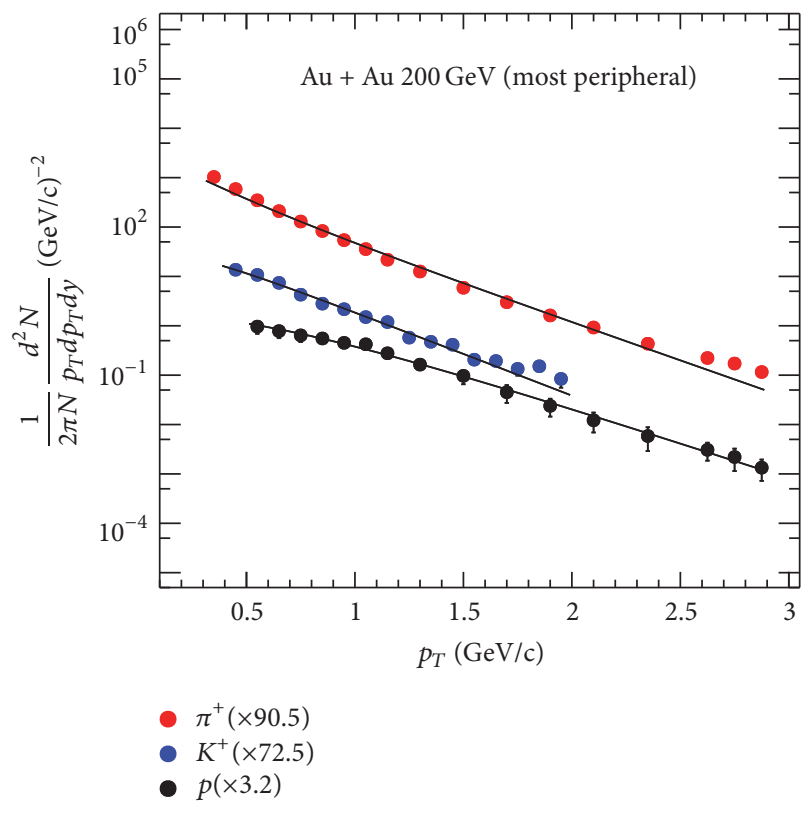

FIGURE 16: Fitting of invariant $p_{T}$ spectra with Tsallis distribution including radial flow using (8) for $\pi^{+}$[18], $K^{+}$[18], and $p$ [18] in most peripheral $\mathrm{Au}+\mathrm{Au}$ collisions at $\sqrt{s_{\mathrm{NN}}}=200 \mathrm{GeV}$.

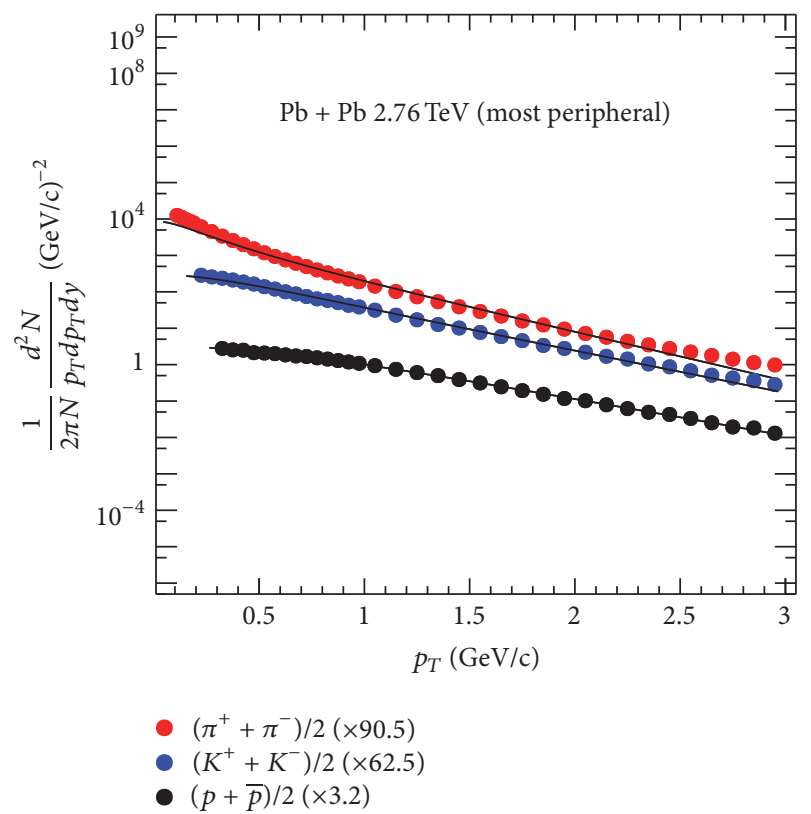

FIgURE 17: Fitting of invariant $p_{T}$ spectra with Tsallis distribution including radial flow using (8) for $\left(\pi^{+}+\pi^{-}\right) / 2[19,20],\left(K^{+}+K^{-}\right) / 2$ $[19,20]$, and $(p+\bar{p}) / 2[19,20]$ in most peripheral $\mathrm{Pb}+\mathrm{Pb}$ collisions at $\sqrt{s_{\mathrm{NN}}}=2.76 \mathrm{TeV}$; symbols represent the data points and the line is the fit function.

\section{Acknowledgments}

Dhananjaya Thakur acknowledges the financial support from UGC, New Delhi, and Sushanta Tripathy acknowledges the DST-INSPIRE scheme of Government of India for financial support.

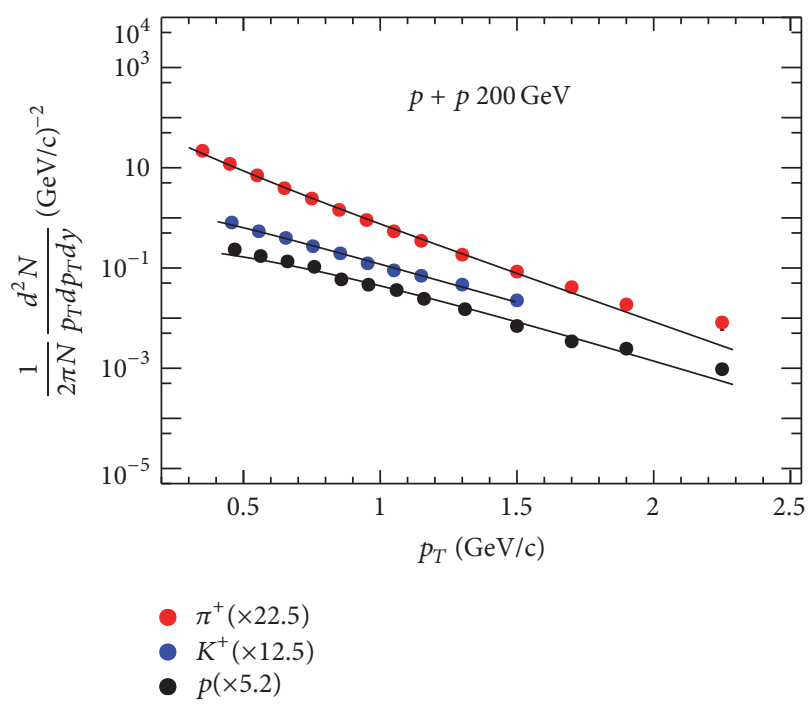

FIgURE 18: Fitting of invariant $p_{T}$ spectra with Tsallis distribution including radial flow using (8) for $\pi^{+}[21], K^{+}[21]$, and $p$ [21] in $p+p$ collisions at $\sqrt{s_{\mathrm{NN}}}=200 \mathrm{GeV}$. Symbols represent the data points and the line is the fit function.

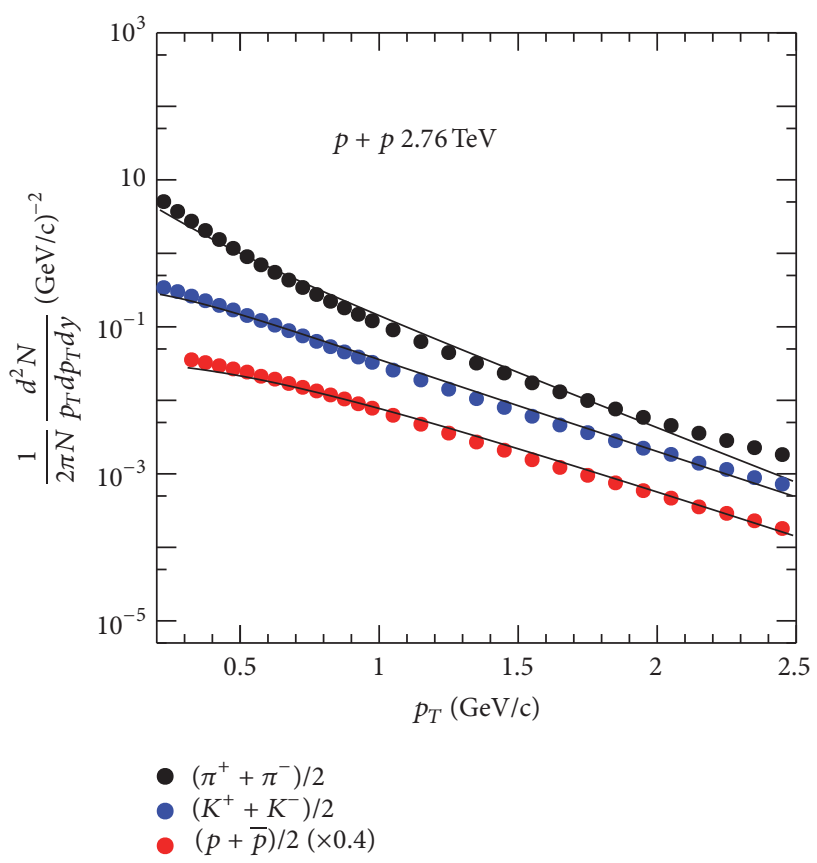

FIGURE 19: Fitting of invariant $p_{T}$ spectra with Tsallis distribution including radial flow using (8) for $\left(\pi^{+}+\pi^{-}\right) / 2[19,20],\left(K^{+}+K^{-}\right) / 2$ $[19,20]$, and $(p+\bar{p}) / 2[19,20]$ in $p+p$ collisions at $\sqrt{s_{\mathrm{NN}}}=2.76 \mathrm{TeV}$. Symbols represent the data points and the line is the fit function.

\section{References}

[1] R. Hagedorn, "Statistical thermodynamics of strong interactions at high energies," Nuovo Cimento Supplements, vol. 3, pp. 147-186, 1965.

[2] C. Michael and L. Vanryckeghem, "Consequences of momentum conservation for particle production at large transverse momentum," Journal of Physics G: Nuclear Physics, vol. 3, no. 8, pp. L151-L156, 1977. 
[3] C. Michael, "Large transverse momentum and large mass productioin in hadronic interactions," Progress in Particle and Nuclear Physics, vol. 2, pp. 1-39, 1979.

[4] G. Arnison, A. Astbury, B. Aubert et al., "Transverse momentum spectra for charged particles at the CERN protonantiproton collider," Physics Letters B, vol. 118, no. 1-3, pp. 167$172,1982$.

[5] R. Hagedorn, "Multiplicities, $p_{T}$ distributions and the expected hadron $\rightarrow$ quark-gluon phase transition," La Rivista del Nuovo Cimento, vol. 6, no. 10, pp. 1-50, 1983.

[6] B. I. Abelev and STAR Collaboration, "Strange particle production in $p+p$ collisions at $\sqrt{s}=200 \mathrm{GeV}$," Physical Review C, vol. 75, no. 6, Article ID 064901, 21 pages, 2007.

[7] A. Adare and PHENIX Collaboration, "Identified charged hadron production in $p+p$ collisions at $\sqrt{s}=200$ and $62.4 \mathrm{GeV}$," Physical Review C, vol. 83, no. 6, Article ID 064903, 2011.

[8] K. Aamodt and ALICE Collaboration, "Production of pions, kaons and protons in pp collisions at $\sqrt{s}=900 \mathrm{GeV}$ with ALICE at the LHC," The European Physical Journal C, vol. 71, article 1655, 2011.

[9] B. Abelev and ALICE Collaboration, "Neutral pion and $\eta$ meson production in proton-proton collisions at $\sqrt{s}=0.9$ and $\sqrt{s}=$ 7 TeV," Physics Letters B, vol. 717, no. 1-3, pp. 162-172, 2012.

[10] B. Abelev and ALICE Collaboration, "Multi-strange baryon production in pp collisions at $\sqrt{s}=7 \mathrm{TeV}$ with ALICE," Physics Letters B, vol. 712, no. 4-5, pp. 309-318, 2012.

[11] S. Chatrchyan and CMS Collaboration, "Study of the inclusive production of charged pions, kaons, and protons in pp collisions at $\sqrt{s}=0.9,2.76$ and $7 \mathrm{TeV}$,' The European Physical Journal C, vol. 72, article 2164, 2012.

[12] T. Bhattacharyya, P. Garg, R. Sahoo, and P. Samantray, "Time evolution of temperature fluctuation in a non-equilibrated system," The European Physical Journal A, vol. 52, article 283, 2016.

[13] C. Tsallis, "Possible generalization of Boltzmann-Gibbs statistics," Journal of Statistical Physics, vol. 52, no. 1-2, pp. 479-487, 1988.

[14] C. Tsallis, "Nonadditive entropy: the concept and its use," The European Physical Journal A, vol. 40, no. 3, pp. 257-266, 2009.

[15] C. Tsallis, Introduction to Nonextensive Statistical Mechanics, Springer, New York, NY, USA, 2009.

[16] J. Cleymans and D. Worku, "The Tsallis distribution in protonproton collisions at $\sqrt{s}=0.9 \mathrm{TeV}$ at the LHC," Journal of Physics G: Nuclear Physics, vol. 39, no. 2, Article ID 025006, 2012.

[17] M. Rybczyński and Z. Włodarczyk, "Tsallis statistics approach to the transverse momentum distributions in p-p collisions," The European Physical Journal C, vol. 74, article 2785, 2014.

[18] S. S. Adler and PHENIX Collaboration, "Identified charged particle spectra and yields in $\mathrm{Au}+\mathrm{Au}$ collisions at $\sqrt{\boldsymbol{s}_{N N}}=$ 200 GeV," Physical Review C, vol. 69, no. 3, Article ID 034909 , 2004.

[19] B. Abelev and ALICE Collaboration, "Production of charged pions, kaons and protons at large transverse momenta in $\mathrm{pp}$ and $\mathrm{Pb}-\mathrm{Pb}$ collisions at $\sqrt{\boldsymbol{s}_{\mathrm{NN}}}=2.76 \mathrm{TeV}$," Physics Letters B, vol. 736, pp. 196-207, 2014.

[20] B. Abelev and ALICE Collaboration, " $K_{S}^{0}$ and $\Lambda$ production in $\mathrm{Pb}-\mathrm{Pb}$ collisions at $\sqrt{s_{N N}}=2.76 \mathrm{TeV}$," Physical Review Letters, vol. 111, no. 22, Article ID 222301, 2013.

[21] J. Adams and STAR Collaboration, "Pion, kaon, proton and anti-proton transverse momentum distributions from $\mathrm{p}+\mathrm{p}$ and $\mathrm{d}+$ Au collisions at $\sqrt{s_{N N}}=200 \mathrm{GeV}$," Physics Letters B, vol. 616, no. 1-2, pp. 8-16, 2005.
[22] D. K. Mishra, P. Garg, P. K. Netrakanti, and A. K. Mohanty, "Netbaryon number fluctuations with the hadron resonance gas model using Tsallis distribution," Journal of Physics G: Nuclear Physics, vol. 42, no. 10, Article ID 105105, 2015.

[23] P. Sett and P. Shukla, "Inferring freeze-out parameters from pion measurements at RHIC and LHC," International Journal of Modern Physics E, vol. 24, no. 6, Article ID 1550046, 2015.

[24] T. Bhattacharyya, J. Cleymans, A. Khuntia, P. Pareek, and R. Sahoo, "Radial flow in non-extensive thermodynamics and study of particle spectra at LHC in the limit of small $(q-1)$," The European Physical Journal A, vol. 52, article 30, 2016.

[25] H. Zheng and L. Zhu, "Can Tsallis distribution fit all the particle spectra produced at RHIC and LHC?” Advances in High Energy Physics, vol. 2015, Article ID 180491, 9 pages, 2015.

[26] Z. Tang, Y. Xu, L. Ruan, G. van Buren, F. Wang, and Z. Xu, "Spectra and radial flow in relativistic heavy ion collisions with Tsallis statistics in a blast-wave description," Physical Review C, vol. 79, no. 5, Article ID 051901, 5 pages, 2009.

[27] B. De, "Non-extensive statistics and understanding particle production and kinetic freeze-out process from $p_{T}$-spectra at 2.76 TeV," The European Physical Journal A, vol. 50, article 138, 2014.

[28] I. Bediaga, E. M. F. Curado, and J. M. De Miranda, "Nonextensive thermodynamical equilibrium approach in $e^{+} e^{-} \rightarrow$ hadrons," Physica A: Statistical Mechanics and Its Applications, vol. 286, no. 1, pp. 156-163, 2000.

[29] S. Tripathy, T. Bhattacharyya, P. Garg, P. Kumar, R. Sahoo, and J. Cleymans, "Nuclear modification factor using Tsallis nonextensive statistics," The European Physical Journal A, vol. 52, no. 9, article 289, 2016.

[30] A. Khuntia, P. Sahoo, P. Garg, R. Sahoo, and J. Cleymans, "Speed of sound in hadronic matter using non-extensive Tsallis statistics," The European Physical Journal A, vol. 52, article 292, 2016.

[31] G. Wilk and Z. Włodarczyk, "Quasi-power law ensembles," Acta Physica Polonica B, vol. 46, no. 6, pp. 1103-1122, 2015.

[32] K. Ürmössy, G. G. Barnaföldi, and T. S. Biró, "Generalised Tsallis statistics in electron-positron collisions," Physics Letters $B$, vol. 701, no. 1, pp. 111-116, 2011.

[33] K. Ürmössy, G. G. Barnaföldi, and T. S. Biró, "Microcanonical jet-fragmentation in proton-proton collisions at LHC energy," Physics Letters B, vol. 718, no. 1, pp. 125-129, 2012.

[34] P. K. Khandai, P. Sett, P. Shukla, and V. Singh, "Hadron spectra IN $p+p$ collisions at RHIC AND LHC energies," International Journal of Modern Physics A, vol. 28, no. 16, Article ID 1350066, 2013.

[35] B.-C. Li, Y.-Z. Wang, and F.-H. Liu, "Formulation of transverse mass distributions in $\mathrm{Au}-\mathrm{Au}$ collisions at $\sqrt{s_{N N}}=$ 200 GeV/nucleon," Physics Letters B, vol. 725, no. 4-5, pp. 352356, 2013.

[36] L. Marques, J. Cleymans, and A. Deppman, "Description of high-energy $p p$ collisions using Tsallis thermodynamics: transverse momentum and rapidity distributions," Physical Review D, vol. 91, no. 5, Article ID 054025, 2015.

[37] A. Adare and PHENIX Collaboration, "Measurement of neutral mesons in $p+p$ collisions at $\sqrt{s}=200 \mathrm{GeV}$ and scaling properties of hadron production," Physical Review D, vol. 83, no. 5, Article ID 052004, 2011.

[38] A. Adare and PHENIX Collaboration, "Identified charged hadron production in $p+p$ collisions at $\sqrt{s}=200$ and $62.4 \mathrm{GeV}$," Physical Review C, vol. 83, no. 6, Article ID 064903, 29 pages, 2011. 
[39] K. Aamodt and ALICE Collaboration, "Transverse momentum spectra of charged particles in proton-proton collisions at $\sqrt{s}=$ $900 \mathrm{GeV}$ with ALICE at the LHC," Physics Letters B, vol. 693, no. 2, pp. 53-68, 2010.

[40] K. Aamodt and ALICE Collaboration, "Production of pions, kaons and protons in pp collisions at $\sqrt{s}=900 \mathrm{GeV}$ with ALICE at the LHC,' The European Physical Journal C, vol. 71, article 1655, 2011.

[41] V. Khachatryan and CMS Collaboration, "Transverse-momentum and pseudorapidity distributions of charged hadrons in pp collisions at 0.9 and $2.36 \mathrm{TeV}$,' Journal of High Energy Physics, vol. 2010, article 41, 2010.

[42] V. Khachatryan and CMS Collaboration, "Transverse-momentum and pseudorapidity distributions of charged hadrons in $p p$ collisions at $\sqrt{s}=7$ TeV," Physical Review Letters, vol. 105, Article ID 022002, 2010.

[43] G. Aad and ATLAS Collaboration, "Charged-particle multiplicities in $p p$ interactions measured with the ATLAS detector at the LHC," New Journal of Physics, vol. 13, Article ID 053033, 2011.

[44] B. Abelev and ALICE Collaboration, "Pion, kaon, and proton production in central $\mathrm{Pb}-\mathrm{Pb}$ collisions at $\sqrt{{s_{N N}}}=2.76 \mathrm{TeV}$," Physical Review Letters, vol. 109, no. 25, Article ID 252301, 11 pages, 2012.

[45] J. Adams and STAR Collaboration, "Experimental and theoretical challenges in the search for the quark-gluon plasma: the STAR Collaboration's critical assessment of the evidence from RHIC collisions," Nuclear Physics A, vol. 757, no. 1-2, pp. 102183, 2005.

[46] J. Cleymans and M. D. Azmi, “The Tsallis distribution at large transverse momenta," The European Physical Journal C, vol. 75, article 430, 2015.

[47] B.-C. Li, Z. Zhang, J.-H. Kang, G.-X. Zhang, and F.-H. Liu, "Tsallis statistical interpretation of transverse momentum spectra in high-energy $p \mathrm{~A}$ collisions," Advances in High Energy Physics, vol. 2015, Article ID 741816, 10 pages, 2015.

[48] C. Beck, "Non-extensive statistical mechanics and particle spectra in elementary interactions," Physica A: Statistical Mechanics and Its Applications, vol. 286, no. 1, pp. 164-180, 2000.

[49] C. Ristea, A. Jipa, O. Ristea et al., "Study of the multiplicity distributions in relativistic nucleus-nucleus collisions using the multiplicity distribution moments method," EPJ Web of Conferences, vol. 66, Article ID 04024, 4 pages, 2014.

[50] V.5.34/32, CERN ROOT, 2015, http://root.cern.ch.

[51] H.-R. Wei, F.-H. Liu, and R. A. Lacey, "Disentangling random thermal motion of particles and collective expansion of source from transverse momentum spectra in high energy collisions," https://arxiv.org/abs/1509.09083.

[52] S. Chatterjee, S. Das, L. Kumar et al., "Freeze-out parameters in heavy-ion collisions at AGS, SPS, RHIC, and LHC energies," Advances in High Energy Physics, vol. 2015, Article ID 349013, 20 pages, 2015.

[53] S. Chatterjee, B. Mohanty, and R. Singh, "Freezeout hypersurface at energies available at the CERN Large Hadron Collider from particle spectra: flavor and centrality dependence," Physical Review C, vol. 92, no. 2, Article ID 024917, 10 pages, 2015.

[54] C. M. Hung and E. Shuryak, "Equation of state, radial flow, and freeze-out in high energy heavy ion collisions," Physical Review C, vol. 57, no. 4, pp. 1891-1906, 1998.

[55] H. Zheng and L. Zhu, "Comparing the Tsallis distribution with and without thermodynamical description in $p+p$ collisions," Advances in High Energy Physics, vol. 2016, Article ID 9632126, 10 pages, 2016.
[56] M. Biyajima, T. Mizoguchi, N. Nakajima, N. Suzuki, and G. Wilk, "Modified Hagedorn formula including temperature fluctuation: estimation of temperatures at RHIC experiments," The European Physical Journal C, vol. 48, no. 2, pp. 597-603, 2006.

[57] T. S. Biró, G. G. Barnaföldi, and P. Ván, "New entropy formula with fluctuating reservoir," Physica A: Statistical Mechanics and Its Applications, vol. 417, pp. 215-220, 2015.

[58] T. S. Biró, P. Ván, G. G. Barnaföldi, and K. Ürmössy, "Statistical power law due to reservoir fluctuations and the Universal Thermostat Independence principle," Entropy, vol. 16, no. 12, pp. 6497-6514, 2014.

[59] H.-L. Lao, H.-R. Wei, F.-H. Liu, and R. A. Lacey, "An evidence of mass-dependent differential kinetic freeze-out scenario observed in $\mathrm{Pb}-\mathrm{Pb}$ collisions at $2.76 \mathrm{TeV}$," The European Physical Journal A, vol. 52, no. 7, article 203, 2016. 

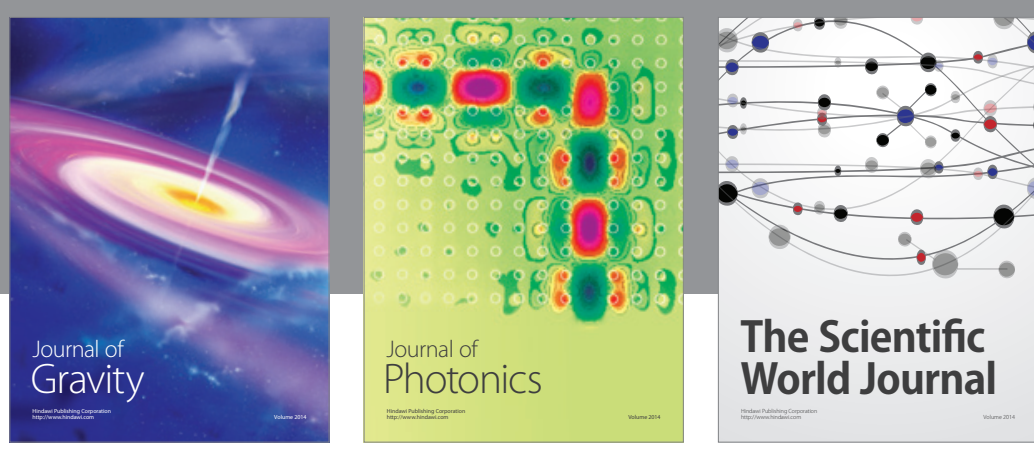

The Scientific World Journal
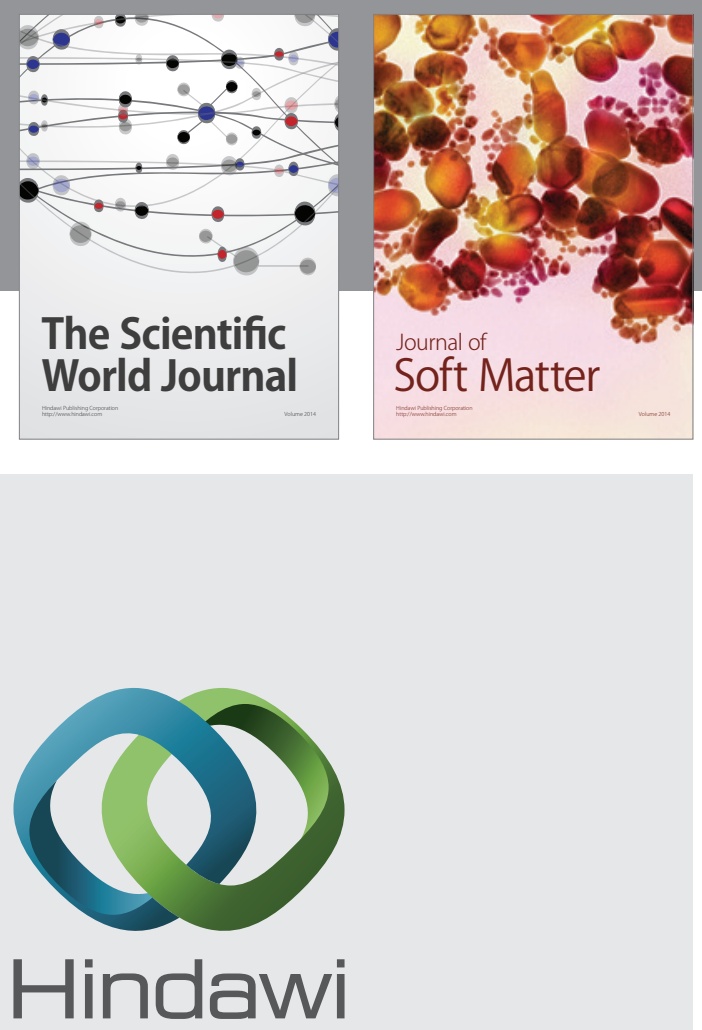

Submit your manuscripts at

http://www.hindawi.com

nternational Journal of

Statistical Mechanics
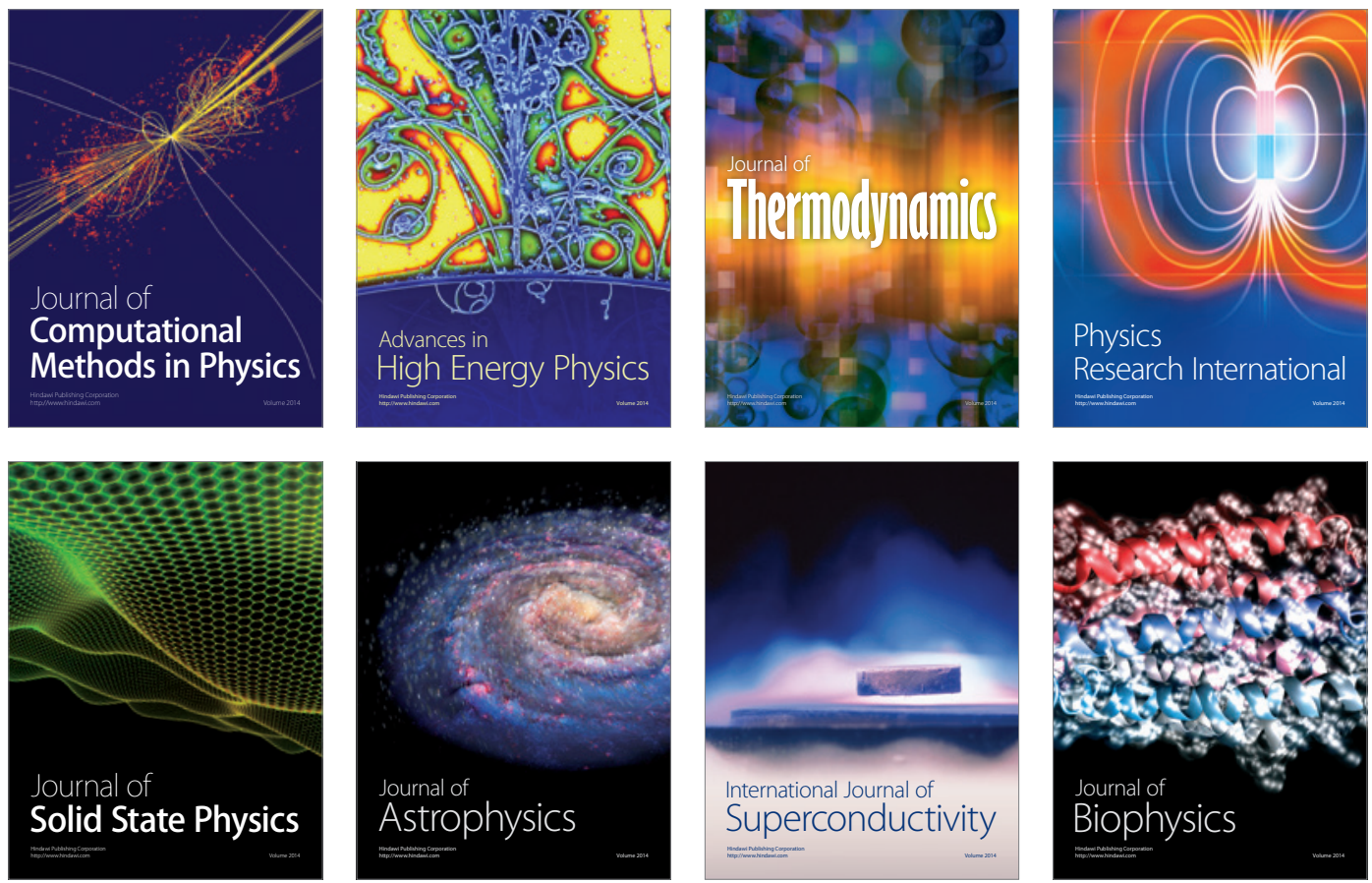
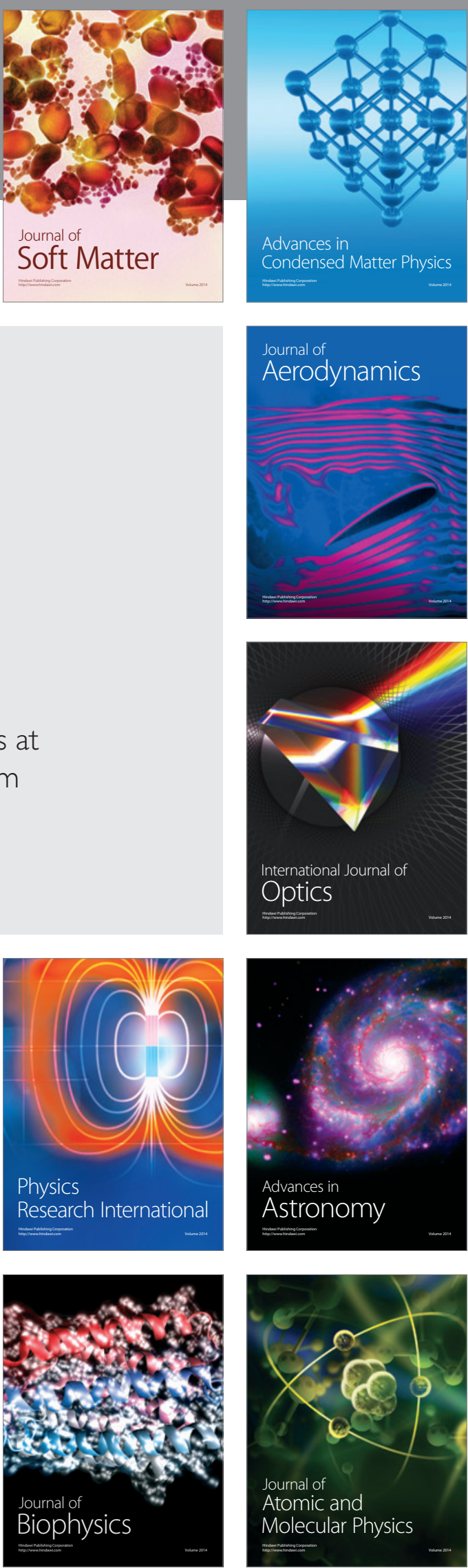